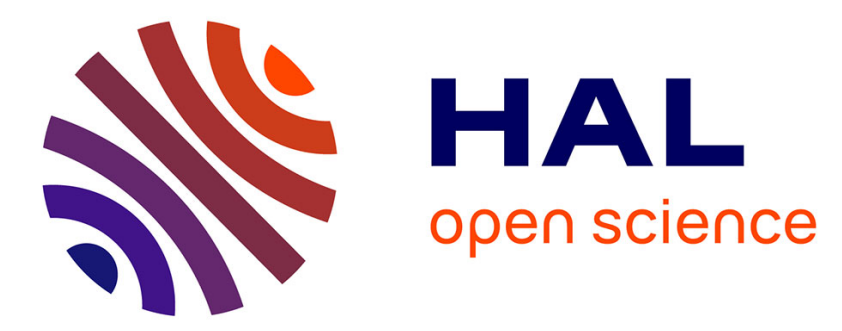

\title{
MASS THRESHOLD FOR INFINITE-TIME BLOWUP IN A CHEMOTAXIS MODEL WITH SPLIT POPULATION
}

\author{
Philippe Laurençot, Christian Stinner
}

\section{> To cite this version:}

Philippe Laurençot, Christian Stinner. MASS THRESHOLD FOR INFINITE-TIME BLOWUP IN A CHEMOTAXIS MODEL WITH SPLIT POPULATION. SIAM Journal on Mathematical Analysis, 2020, 53 (3), pp.3385-3419. hal-02943355v2

\section{HAL Id: hal-02943355 \\ https://hal.science/hal-02943355v2}

Submitted on 28 Sep 2021

HAL is a multi-disciplinary open access archive for the deposit and dissemination of scientific research documents, whether they are published or not. The documents may come from teaching and research institutions in France or abroad, or from public or private research centers.
L'archive ouverte pluridisciplinaire HAL, est destinée au dépôt et à la diffusion de documents scientifiques de niveau recherche, publiés ou non, émanant des établissements d'enseignement et de recherche français ou étrangers, des laboratoires publics ou privés. 


\title{
MASS THRESHOLD FOR INFINITE-TIME BLOWUP IN A CHEMOTAXIS MODEL WITH SPLIT POPULATION
}

\author{
PHILIPPE LAURENÇOT AND CHRISTIAN STINNER
}

Abstract. We study the chemotaxis model

$$
\begin{aligned}
\partial_{t} u & =\operatorname{div}(\nabla u-u \nabla w)+\theta v-u & & \text { in }(0, \infty) \times \Omega, \\
\partial_{t} v & =u-\theta v & & \text { in }(0, \infty) \times \Omega, \\
\partial_{t} w & =D \Delta w-\alpha w+v & & \text { in }(0, \infty) \times \Omega,
\end{aligned}
$$

with no-flux boundary conditions in a bounded and smooth domain $\Omega \subset \mathbb{R}^{2}$, where $u$ and $v$ represent the densities of subpopulations of moving and static individuals of some species, respectively, and $w$ the concentration of a chemoattractant. We prove that, in an appropriate functional setting, all solutions exist globally in time. Moreover, we establish the existence of a critical mass $M_{c}>0$ of the whole population $u+v$ such that, for $M \in\left(0, M_{c}\right)$, any solution is bounded, while, for almost all $M>M_{c}$, there exist solutions blowing up in infinite time. The building block of the analysis is the construction of a Liapunov functional. As far as we know, this is the first result of this kind when the mass conservation includes the two subpopulations and not only the moving one.

\section{INTRODUCTION}

We investigate the dynamics of a chemotaxis model describing the space and time evolution of a species including moving and static individuals, as well as that of a chemoattractant produced by the latter. More precisely, on the one hand, the motion of moving individuals is due to diffusion with a bias towards regions of high concentrations of the chemoattractant. On the other hand, the chemoattractant is produced only by the static individuals, while its spatial fluctuations result from standard diffusion. Finally, the total population in the species is assumed to be constant throughout time evolution, with a linear exchange between the two subpopulations. Denoting the densities of moving and static individuals by $u$ and $v$, respectively, and the concentration of chemoattractant by $w$, the mathematical model reads, after a suitable rescaling of the parameters,

$$
\begin{aligned}
\partial_{t} u & =\operatorname{div}(\nabla u-u \nabla w)+\theta v-u & & \text { in }(0, \infty) \times \Omega, \\
\partial_{t} v & =u-\theta v & & \text { in }(0, \infty) \times \Omega, \\
\partial_{t} w & =D \Delta w-\alpha w+v & & \text { in }(0, \infty) \times \Omega,
\end{aligned}
$$

Date: September 28, 2021.

2010 Mathematics Subject Classification. 35B40, 35B44, 35M33, 35K10, 35Q92, 92C17.

Key words and phrases. Chemotaxis system, species with two subpopulations, global solutions, critical mass, infinite-time blowup. 
supplemented with no-flux boundary conditions

$$
\nabla u \cdot \mathbf{n}=\nabla w \cdot \mathbf{n}=0 \quad \text { on }(0, \infty) \times \partial \Omega
$$

and initial conditions

$$
(u, v, w)(0)=\left(u_{0}, v_{0}, w_{0}\right) \quad \text { in } \Omega .
$$

We assume that $\Omega \subset \mathbb{R}^{2}$ is a bounded domain with smooth boundary and the constants $D$, $\alpha$, and $\theta$ are positive.

The chemotaxis mechanism described by the system (1.1) differs from the classical Keller-Segel chemotaxis system which involves only a single population of individuals besides a chemotactic attractant and reads

$$
\begin{aligned}
& \partial_{t} u=\operatorname{div}(\nabla u-u \nabla w) \\
& \text { in }(0, \infty) \times \Omega \text {, } \\
& \partial_{t} w=D \Delta w-\alpha w+\theta^{-1} u \\
& \text { in }(0, \infty) \times \Omega \text {, }
\end{aligned}
$$

supplemented with no-flux boundary conditions. Indeed, in (1.1), the species is split in two subpopulations with different characteristics. On the one hand, only a subpopulation of the species under consideration (the moving individuals $u$ ) is sensitive to the chemotactic attractant $w$. On the other hand, the attractant is produced by the other subpopulation $v$. These two features are actually met in several biological situations and indirect signal production is already included in the original KellerSegel model for slime mold morphogenesis [16], but is also found in ecology $[9,20,26,27,31,32,36]$, as well as in models for tumor invasion [30] and embryogenesis [24], to name but a few (see also the recent review [23] for an extended list of references). Also, we assume in (1.1) that the total population in the species is constant, a feature which is also met in some models in ecology [27] and biofilm formation [28].

The system (1.1) is actually a particular case of a model developed in [27] for foraging ants if we assume additionally that the food is homogeneously scattered throughout the domain and the motion of ants is only biased by the attractant and not by the spatial environment. The system (1.1) can also be seen as a variant of chemotaxis models derived in $[9,20,26,30,31,36]$ with different interpretations of the species and its two subpopulations and some modifications in the kinetics. In [9], it is some building material (such as soil) which is, either carried by insects, or deposited on the ground, and we replace a source of carrying insects by the assumption that the quantity of building material is constant during time evolution. With the same modification, the system (1.1) is closely related to models for the spreading of mountain pine beetles which are derived and studied in $[20,26,31,36]$, dividing the population into flying and nesting beetles. Finally, proliferation of cancer cells is considered in [30], separating migrating cells from proliferating cells, and (1.1) fits into the general framework developed in [30] but with a different dynamics for the attractant.

As far as mathematical analysis is concerned, the model introduced in [9], which is also a simplified variant of the models in $[26,31,36]$, is the subject of a number of analytical results dealing with the global existence of solutions and the asymptotic behavior of bounded solutions, see, e.g., [14,18,22,35]. In all these results, instead of the splitting term $\theta v-u$ in (1.1a), the corresponding models contain in the taxis equation a dissipative term $f(u)$ depending only on $u$ generalizing the prototype $f(u)=$ 
$1-\mu u$. In case of $f \equiv 0$, a critical mass phenomenon for global solutions is proved in [17,34]. For the model developed in [30], which includes a splitting term similar to $\theta v-u$ in the taxis equation, the global existence of solutions is proved in [30], while further results concerning their large time behavior are lacking.

To the best of our knowledge, our results concerning the behavior of solutions to (1.1)-(1.2) seem to be the first going beyond global existence for a chemotaxis model involving a species divided into moving and static individuals and containing in the taxis equation a splitting term depending on both subpopulations.

Our first result states the global existence and well-posedness for (1.1)-(1.2) in an appropriate functional setting. To this end, for $r \in(1, \infty)$, we set

$$
\begin{aligned}
& W_{r, \mathcal{B}}^{m}(\Omega):=\left\{z \in W_{r}^{m}(\Omega): \nabla z \cdot \mathbf{n}=0 \text { on } \partial \Omega\right\} \quad \text { if } \quad 1+\frac{1}{r}<m \leq 2, \\
& W_{r, \mathcal{B}}^{m}(\Omega):=W_{r}^{m}(\Omega) \quad \text { if } \quad-1+\frac{1}{r}<m<1+\frac{1}{r}, \\
& W_{r, \mathcal{B}}^{m}(\Omega):=W_{r /(r-1)}^{-m}(\Omega)^{\prime} \quad \text { if } \quad-2+\frac{1}{r}<m \leq-1+\frac{1}{r},
\end{aligned}
$$

and

$$
W_{r, \mathcal{B},+}^{m}(\Omega):=\left\{z \in W_{r, \mathcal{B}}^{m}(\Omega): z \geq 0 \text { in } \Omega\right\},
$$

where $W_{r}^{m}(\Omega), m \in[0, \infty), r \in[1, \infty)$, denote the usual Sobolev spaces, see [3, Section 5].

Theorem 1.1. Let $M>0$ and $\left(u_{0}, v_{0}, w_{0}\right) \in W_{3,+}^{1}\left(\Omega ; \mathbb{R}^{3}\right)$ satisfying

$$
\left\|u_{0}+v_{0}\right\|_{L_{1}(\Omega)}=M \text {. }
$$

Then the system (1.1)-(1.2) has a unique nonnegative weak solution $(u, v, w)$ in $W_{3}^{1}$ defined on $[0, \infty)$ satisfying

$$
\begin{aligned}
& u \in C\left([0, \infty) ; W_{3,+}^{1}(\Omega)\right) \cap C^{1}\left([0, \infty) ; W_{3 / 2}^{1}\left(\Omega ; \mathbb{R}^{2}\right)^{\prime}\right), \\
& v \in C^{1}\left([0, \infty) ; W_{3,+}^{1}(\Omega)\right), \\
& w \in C\left([0, \infty) ; W_{3,+}^{1}(\Omega)\right) \cap C^{1}\left([0, \infty) ; W_{3 / 2}^{1}\left(\Omega ; \mathbb{R}^{2}\right)^{\prime}\right),
\end{aligned}
$$

and

$$
\|(u+v)(t)\|_{L_{1}(\Omega)}=M, \quad t \geq 0 .
$$

Moreover,

$$
\begin{aligned}
& u \in C\left((0, \infty) ; W_{3, \mathcal{B}}^{2}(\Omega)\right) \cap C^{1}\left((0, \infty) ; L_{3}(\Omega)\right), \\
& w \in C\left((0, \infty) ; W_{3, \mathcal{B}}^{2}(\Omega)\right) \cap C^{1}\left((0, \infty) ; L_{3}(\Omega)\right) .
\end{aligned}
$$

We next establish a critical mass phenomenon for (1.1)-(1.2). More precisely, we show the existence of a critical mass $M_{c}>0$, where $M_{c}=4 \pi(1+\theta) D$ in the general case and $M_{c}=8 \pi(1+\theta) D$ in the radial setting in a ball, such that all solutions are bounded if the initial mass $M$ satisfies $M<M_{c}$, while solutions blowing up in infinite time exist for almost all $M>M_{c}$.

We begin with the statement of the boundedness result for $M$ being subcritical. 
Theorem 1.2. Let $M>0$ and consider $\left(u_{0}, v_{0}, w_{0}\right) \in \mathcal{I}_{M}$, where

$$
\mathcal{I}_{M}:=\left\{(u, v, w) \in W_{3,+}^{1}(\Omega) \times W_{3,+}^{1}(\Omega) \times W_{2,+}^{2}(\Omega):\|u+v\|_{L_{1}(\Omega)}=M\right\} .
$$

$B y(u, v, w)$ we denote the solution to (1.1)-(1.2) given by Theorem 1.1.

(a) If $M=\left\|u_{0}+v_{0}\right\|_{L_{1}(\Omega)} \in(0,4 \pi(1+\theta) D)$, then

$$
\sup _{t \geq 0}\left\{\|u(t)\|_{L_{\infty}(\Omega)}+\|v(t)\|_{L_{\infty}(\Omega)}+\|w(t)\|_{L_{\infty}(\Omega)}\right\}<\infty .
$$

(b) If $\Omega=B_{R}(0)$ is the ball of radius $R>0$ centered at $x=0,\left(u_{0}, v_{0}, w_{0}\right)$ are radially symmetric, and $M=\left\|u_{0}+v_{0}\right\|_{L_{1}(\Omega)} \in(0,8 \pi(1+\theta) D)$, then (1.8) is also satisfied.

The corresponding unboundedness result for $M$ being supercritical is the following.

Theorem 1.3. Let $M>0$.

(a) If $M \in(4 \pi(1+\theta) D, \infty) \backslash(4 \pi(1+\theta) D \mathbb{N})$, then there are solutions $(u, v, w)$ to (1.1)-(1.2) with initial conditions in $\mathcal{I}_{M}$ with an unbounded first component; that is,

$$
\lim _{t \rightarrow \infty}\|u(t)\|_{L_{\infty}(\Omega)}=\infty .
$$

(b) Assume that $\Omega=B_{R}(0)$ for some $R>0$. If $M \in(8 \pi(1+\theta) D, \infty)$, then there are solutions $(u, v, w)$ to (1.1)-(1.2) with radially symmetric initial conditions in $\mathcal{I}_{M}$ with an unbounded first component, i.e., satisfying (1.9).

While for Keller-Segel systems there are many results on critical mass phenomena distinguishing between boundedness and finite-time blowup, to the best of our knowledge such phenomena separating boundedness from infinite-time blowup seem to be scarcer. Still, the latter phenomenon has been established in [8] for a chemotaxis model with volume filling effect as well as in [17,34] for models related to (1.1), but with 0 instead of $\theta v-u$ in the right hand side of (1.1a).

We prove the results presented above by mainly extending the strategy from [17] to (1.1)-(1.2), which in turn has its roots in [11-13]. We start by constructing a Liapunov functional for (1.1)-(1.2) in Section 2, see (2.2), which is of general interest far beyond the results of this work and actually the building block of our analysis. In Section 3 we prove the global existence of solutions to (1.1)-(1.2) in Theorem 1.1 by mainly relying on Amann's theory for partially diffusive parabolic systems in an appropriate functional setting, in conjunction with a series of a priori estimates, some of them involving parts of the Liapunov functional. In Section 4 we prove that global solutions to (1.1)-(1.2) are bounded, provided $M=\left\|u_{0}+v_{0}\right\|_{L_{1}(\Omega)}$ is suitably small, see Theorem 1.2. Here we first prove with the help of the Trudinger-Moser inequality that the Liapunov functional constructed in Section 2 is bounded from below for subcritical $M$ and use this property as a starting point for the derivation of further estimates. Finally, in Section 5 we prove that, for $M$ sufficiently large, unbounded solutions exist, as stated in Theorem 1.3. Here we use the strategy pioneered in $[12,13]$ and further developed in $[17,29]$. On the one hand, we establish that any bounded solution approaches the set of stationary solutions when $t \rightarrow \infty$. On the other hand, we show that the Liapunov functional is bounded from below on the set of steady states with fixed mass $M>M_{c}$, but not bounded from below on the set of initial data with mass $M$. Hence, solutions emanating from initial data for which the Liapunov 
functional takes sufficiently negative values cannot be global and bounded and therefore have to blow up in infinite time. As compared to [17], the Liapunov functional constructed here features additional terms involving $v$, so that some arguments, in particular the proof of blowup, are more involved.

\section{A Liapunov functional}

One of the main contributions of this work is the construction of a Liapunov function for (1.1)(1.2). To this end, we define for $\theta>0$

$$
L(r):=r \ln r-r+1, \quad L_{\theta}(r):=\frac{L(\theta r)}{\theta}=r \ln (\theta r)-r+\frac{1}{\theta}, \quad r \geq 0,
$$

and observe that both functions are nonnegative. We next set

$$
\begin{aligned}
\mathcal{L}(u, v, w):= & \int_{\Omega}\left(L(u)+L_{\theta}(v)-(u+v) w\right) \mathrm{d} x \\
& +\frac{1+\theta}{2}\left(D\|\nabla w\|_{L_{2}(\Omega)}^{2}+\alpha\|w\|_{L_{2}(\Omega)}^{2}\right)+\frac{1}{2}\|D \Delta w-\alpha w+v\|_{L_{2}(\Omega)}^{2},
\end{aligned}
$$

and establish that $\mathcal{L}$ is a Liapunov functional for (1.1). We emphasize that, in constrast to [17], the Liapunov functional depends not only on $u$ and $w$, but also on $v$ through the term $L_{\theta}(v)-v w$. This is obviously related to the fact that the conserved quantity throughout time evolution is $\|u+v\|_{L_{1}(\Omega)}$ instead of $\|u\|_{L_{1}(\Omega)}$. Thus, some arguments in the forthcoming sections are more involved as compared to $[17]$.

Lemma 2.1. Let $M>0$. Consider $\left(u_{0}, v_{0}, w_{0}\right) \in \mathcal{I}_{M}$ and denote the corresponding solution to (1.1)-(1.2) given by Theorem 1.1 by $(u, v, w)$. Then

$$
\frac{\mathrm{d}}{\mathrm{d} t} \mathcal{L}(u, v, w)+\mathcal{D}(u, v, w)=0, \quad t>0,
$$

where $\mathcal{D}$ is nonnegative and defined in (2.5) below.

Proof. It follows from (1.1a), (1.1b), and (1.1d) that

$$
\begin{aligned}
\frac{\mathrm{d}}{\mathrm{d} t} \int_{\Omega}(u \ln u-u-u w) \mathrm{d} x= & \int_{\Omega}(\ln u-w)[\operatorname{div}(\nabla u-u \nabla w)+\theta v-u] \mathrm{d} x \\
& -\int_{\Omega}\left(\partial_{t} v+\theta v\right) \partial_{t} w \mathrm{~d} x \\
= & -\int_{\Omega} u|\nabla(\ln u-w)|^{2} \mathrm{~d} x+\int_{\Omega}(\theta v-u) \ln u \mathrm{~d} x \\
& +\int_{\Omega} w \partial_{t} v \mathrm{~d} x-\int_{\Omega}\left(\partial_{t} v+\theta v\right) \partial_{t} w \mathrm{~d} x .
\end{aligned}
$$


Now, by (1.1c),

$$
\begin{aligned}
\int_{\Omega} w \partial_{t} v \mathrm{~d} x= & \int_{\Omega} w\left(\partial_{t}^{2} w-D \Delta \partial_{t} w+\alpha \partial_{t} w\right) \mathrm{d} x \\
= & \int_{\Omega}\left(\partial_{t}\left(w \partial_{t} w\right)-\left(\partial_{t} w\right)^{2}+D \nabla w \cdot \nabla \partial_{t} w+\alpha w \partial_{t} w\right) \mathrm{d} x \\
= & \frac{\mathrm{d}}{\mathrm{d} t} \int_{\Omega}\left(w \partial_{t} w+\frac{D}{2}|\nabla w|^{2}+\frac{\alpha}{2}|w|^{2}\right) \mathrm{d} x-\left\|\partial_{t} w\right\|_{L_{2}(\Omega)}^{2} \\
= & \frac{\mathrm{d}}{\mathrm{d} t} \int_{\Omega} w(D \Delta w-\alpha w+v) \mathrm{d} x+\frac{\mathrm{d}}{\mathrm{d} t}\left(\frac{D}{2}\|\nabla w\|_{L_{2}(\Omega)}^{2}+\frac{\alpha}{2}\|w\|_{L_{2}(\Omega)}^{2}\right)-\left\|\partial_{t} w\right\|_{L_{2}(\Omega)}^{2} \\
= & \frac{\mathrm{d}}{\mathrm{d} t}\left(\int_{\Omega} v w \mathrm{~d} x-D\|\nabla w\|_{L_{2}(\Omega)}^{2}-\alpha\|w\|_{L_{2}(\Omega)}^{2}+\frac{D}{2}\|\nabla w\|_{L_{2}(\Omega)}^{2}+\frac{\alpha}{2}\|w\|_{L_{2}(\Omega)}^{2}\right) \\
& -\left\|\partial_{t} w\right\|_{L_{2}(\Omega)}^{2} \\
= & -\frac{\mathrm{d}}{\mathrm{d} t}\left(\frac{D}{2}\|\nabla w\|_{L_{2}(\Omega)}^{2}+\frac{\alpha}{2}\|w\|_{L_{2}(\Omega)}^{2}-\int_{\Omega} v w \mathrm{~d} x\right)-\left\|\partial_{t} w\right\|_{L_{2}(\Omega)}^{2} .
\end{aligned}
$$

Using again (1.1c),

$$
\begin{aligned}
-\int_{\Omega} \partial_{t} v \partial_{t} w \mathrm{~d} x & =-\int_{\Omega} \partial_{t} w\left(\partial_{t}^{2} w-D \Delta \partial_{t} w+\alpha \partial_{t} w\right) \mathrm{d} x \\
& =-\frac{1}{2} \frac{\mathrm{d}}{\mathrm{d} t}\left\|\partial_{t} w\right\|_{L_{2}(\Omega)}^{2}-D\left\|\nabla \partial_{t} w\right\|_{L_{2}(\Omega)}^{2}-\alpha\left\|\partial_{t} w\right\|_{L_{2}(\Omega)}^{2}
\end{aligned}
$$

and

$$
\begin{aligned}
-\theta \int_{\Omega} v \partial_{t} w \mathrm{~d} x & =-\theta \int_{\Omega} \partial_{t} w\left(\partial_{t} w-D \Delta w+\alpha w\right) \mathrm{d} x \\
& =-\theta\left\|\partial_{t} w\right\|_{L_{2}(\Omega)}^{2}-\frac{\theta}{2} \frac{\mathrm{d}}{\mathrm{d} t}\left(D\|\nabla w\|_{L_{2}(\Omega)}^{2}+\alpha\|w\|_{L_{2}(\Omega)}^{2}\right) .
\end{aligned}
$$

Gathering the above four identities implies

$$
\begin{aligned}
\frac{\mathrm{d}}{\mathrm{d} t} \int_{\Omega} & (u \ln u-u-(u+v) w) \mathrm{d} x+\frac{1+\theta}{2} \frac{\mathrm{d}}{\mathrm{d} t}\left(D\|\nabla w\|_{L_{2}(\Omega)}^{2}+\alpha\|w\|_{L_{2}(\Omega)}^{2}\right)+\frac{1}{2} \frac{\mathrm{d}}{\mathrm{d} t}\left\|\partial_{t} w\right\|_{L_{2}(\Omega)}^{2} \\
=- & \int_{\Omega} u|\nabla(\ln u-w)|^{2} \mathrm{~d} x+\int_{\Omega}(\theta v-u) \ln u \mathrm{~d} x \\
& \quad-D\left\|\nabla \partial_{t} w\right\|_{L_{2}(\Omega)}^{2}-(1+\theta+\alpha)\left\|\partial_{t} w\right\|_{L_{2}(\Omega)}^{2} .
\end{aligned}
$$

Finally, since

$$
\begin{aligned}
\frac{\mathrm{d}}{\mathrm{d} t} \int_{\Omega}(v \ln (\theta v)-v) \mathrm{d} x & =\int_{\Omega} \ln (\theta v) \partial_{t} v \mathrm{~d} x \\
& =\int_{\Omega} \ln (\theta v)(u-\theta v) \mathrm{d} x
\end{aligned}
$$


by $(1.1 b)$, we end up with

where

$$
\frac{\mathrm{d}}{\mathrm{d} t} \mathcal{L}(u, v, w)+\mathcal{D}(u, v, w)=0
$$

$$
\begin{aligned}
\mathcal{D}(u, v, w):= & \int_{\Omega} u|\nabla(\ln u-w)|^{2} \mathrm{~d} x+\int_{\Omega}(\theta v-u)(\ln (\theta v)-\ln u) \mathrm{d} x \\
& \quad+D\|\nabla(D \Delta w-\alpha w+v)\|_{L_{2}(\Omega)}^{2}+(1+\theta+\alpha)\|D \Delta w-\alpha w+v\|_{L_{2}(\Omega)}^{2} .
\end{aligned}
$$

Observe that the monotonicity of the logarithm function ensures the nonnegativity of $\mathcal{D}$.

A useful consequence of the availability of a Liapunov functional is the stabilization of global solutions which are bounded in a suitable functional space; that is, the cluster points of such solutions as $t \rightarrow \infty$ in an appropriate topology are necessarily stationary solutions. In that direction, we report the following result, which is similar to [10, Theorem 5.2], [12, Lemma 1], and [17, Proposition 3.8].

Proposition 2.2. Let $M>0$. Consider $\left(u_{0}, v_{0}, w_{0}\right) \in \mathcal{I}_{M}$ and denote the corresponding solution to (1.1)-(1.2) given by Theorem 1.1 by $(u, v, w)$. Assume also that

$$
\sup _{t \geq 0}\|u(t)\|_{L_{\infty}(\Omega)}<\infty
$$

Then there are a sequence $\left(t_{j}\right)_{j \geq 1}$ of positive times, $t_{j} \rightarrow \infty$, and nonnegative functions $\left(u_{*}, v_{*}, w_{*}\right) \in$ $L_{\infty}\left(\Omega ; \mathbb{R}^{2}\right) \times W_{2, \mathcal{B}}^{2}(\Omega)$ such that

$$
\lim _{j \rightarrow \infty}\left[\left\|u\left(t_{j}\right)-u_{*}\right\|_{L_{2}(\Omega)}+\left\|v\left(t_{j}\right)-v_{*}\right\|_{L_{2}(\Omega)}+\left\|w\left(t_{j}\right)-w_{*}\right\|_{W_{2}^{1}(\Omega)}\right]=0
$$

and

$$
\mathcal{L}\left(u_{*}, v_{*}, w_{*}\right) \leq \liminf _{j \rightarrow \infty} \mathcal{L}\left(u\left(t_{j}\right), v\left(t_{j}\right), w\left(t_{j}\right)\right)
$$

where

$$
u_{*}=\frac{\theta M}{\theta+1} \frac{e^{w_{*}}}{\left\|e^{w_{*}}\right\|_{L_{1}(\Omega)}}, \quad v_{*}=\frac{M}{\theta+1} \frac{e^{w_{*}}}{\left\|e^{w_{*}}\right\|_{L_{1}(\Omega)}},
$$

and $w_{*}$ is a solution to the nonlocal elliptic problem

$$
-D \Delta w_{*}+\alpha w_{*}=\frac{M}{\theta+1} \frac{e^{w_{*}}}{\left\|e^{w_{*}}\right\|_{L_{1}(\Omega)}} \quad \text { in } \quad \Omega, \quad \nabla w_{*} \cdot \mathbf{n}=0 \quad \text { on } \quad \partial \Omega .
$$

Proof. The proof is similar to that of [17, Proposition 3.8], after observing that (1.1b), (2.6), and the positivity of $\theta$ imply that

$$
\sup _{t \geq 0}\|v(t)\|_{L_{\infty}(\Omega)}<\infty
$$

while (1.1c), (1.1d), (1.6), and the positivity of $\alpha$ ensure that

$$
\sup _{t \geq 0}\|w(t)\|_{L_{1}(\Omega)}<\infty
$$

Thanks to (2.6), (2.8), and (2.9), we may argue as in [17, Proposition 3.8] to derive first a lower bound on $\mathcal{L}(u, v, w)$ and then the claimed stabilization. 


\section{Global existence}

This section is devoted to the proof of Theorem 1.1 and includes three steps: we first establish the local well-posedness of (1.1)-(1.2) in a suitable functional setting and study the regularity of the solution for positive times. We next derive a series of estimates which excludes the occurrence of finite time blowup.

3.1. Local well-posedness. We start with the local well-posedness of (1.1)-(1.2), which is a consequence of $[2$, Theorem 6.4].

Proposition 3.1. Let $\left(u_{0}, v_{0}, w_{0}\right) \in W_{3}^{1}\left(\Omega ; \mathbb{R}^{3}\right)$ be nonnegative. Then the system (1.1)-(1.2) has a unique nonnegative weak solution $(u, v, w)$ in $W_{3}^{1}$ defined on a maximal time interval $\left[0, T_{m}\right)$, with $T_{m} \in(0, \infty]$, satisfying

$$
\begin{aligned}
(u, w) & \in C\left(\left[0, T_{m}\right) ; W_{3}^{1}\left(\Omega ; \mathbb{R}^{2}\right)\right) \cap C^{1}\left(\left[0, T_{m}\right) ; W_{3 / 2}^{1}\left(\Omega ; \mathbb{R}^{2}\right)^{\prime}\right), \\
v & \in C^{1}\left(\left[0, T_{m}\right) ; W_{3}^{1}(\Omega)\right),
\end{aligned}
$$

and

$$
\|(u+v)(t)\|_{L_{1}(\Omega)}=M=\left\|u_{0}+v_{0}\right\|_{L_{1}(\Omega)}, \quad t \in\left[0, T_{m}\right) .
$$

Furthermore, if there is $T>0$ such that

$$
(u, v, w) \in B U C\left([0, T] \cap\left[0, T_{m}\right) ; W_{3}^{1}\left(\Omega ; \mathbb{R}^{3}\right)\right),
$$

then necessarily $T_{m} \geq T$.

Proof. Throughout the proof, $C$ denotes a positive constant that may vary from line to line and depends only on $\Omega, \theta, D, \alpha,\left\|u_{0}\right\|_{W_{3}^{1}(\Omega)},\left\|v_{0}\right\|_{W_{3}^{1}(\Omega)}$, and $\left\|w_{0}\right\|_{W_{3}^{1}(\Omega)}$. The existence and uniqueness of a weak solution $(u, v, w)$ to (1.1)-(1.2) having the claimed properties, except for nonnegativity and (3.1), rely on [2, Theorem 6.4] and are proved in a completely similar way as [17, Proposition 2.1]. The proof relies on the framework developed in $[2,3]$ to handle systems coupling parabolic equations and ordinary differential equations. Specifically, we set $U=\left(u_{1}, u_{2}, u_{3}\right):=(u, w, v), U^{1}:=(u, w)$, $U^{2}:=v$, and define

$$
A(V):=\left(\begin{array}{cc}
1 & -v_{1} \\
0 & D
\end{array}\right), \quad S^{1}(V):=\left(\begin{array}{c}
\theta v_{3}-v_{1} \\
v_{3}-\alpha v_{2}
\end{array}\right), \quad S^{2}(V):=\left(v_{1}-\theta v_{3}\right)
$$

for $V=\left(v_{1}, v_{2}, v_{3}\right)$. Introducing the operators

$$
\begin{aligned}
& \mathcal{A}^{1}(V) U^{1}:=-\sum_{j=1}^{2} \partial_{j}\left(A(V) \partial_{j} U^{1}\right), \quad \mathcal{B}^{1}(V) U^{1}:=\sum_{j=1}^{2} A(V) \mathbf{n} \cdot \nabla U^{1}, \\
& \mathcal{A}^{2}(V) U^{2}:=0, \quad \mathcal{B}^{2}(V) U^{2}:=0,
\end{aligned}
$$

the system (1.1)-(1.2) can be recast as

$$
\begin{aligned}
\partial_{t} U^{1}+\mathcal{A}^{1}(U) U^{1}+\mathcal{A}^{2}(U) U^{2}=S^{1}(U) & \text { in } \quad(0, \infty) \times \Omega, \\
\partial_{t} U^{2}=S^{2}(U) & \text { in } \quad(0, \infty) \times \Omega,
\end{aligned}
$$




$$
\begin{aligned}
\mathcal{B}^{1}(U) U^{1}+\mathcal{B}^{2}(U) U^{2} & =0 \text { on }(0, \infty) \times \partial \Omega, \\
U(0) & =\left(u_{0}, w_{0}, v_{0}\right) \text { in } \Omega,
\end{aligned}
$$

and its well-posedness, as stated in Proposition 3.1, follows from [2, Theorem 6.4]. Indeed, this result can be applied here since $\left(\mathcal{A}^{1}, \mathcal{B}^{1}\right)$ is normally elliptic by [2, Remarks 4.1 (a)-(iii)] and [2, Condition (6.1)] is satisfied. Denoting the solution to the above system provided by [2, Theorem 6.4] by $U=\left(U^{1}, U^{2}\right)$, we set $(u, w):=U^{1}$ and $v:=U^{2}$ and thereby obtain Proposition 3.1, but yet without the nonnegativity of $(u, v, w)$ and the mass conservation (3.1).

To prove the former, we first notice that, since $W_{3}^{11 / 6}(\Omega)$ embeds continuously in $W_{\infty}^{1}(\Omega)$ by $[25$, Chapter 7, Theorem 1.2] and

$$
\begin{aligned}
& \left(L_{3}(\Omega), W_{3, \mathcal{B}}^{2}(\Omega)\right)_{11 / 12,3} \doteq W_{3, \mathcal{B}}^{11 / 6}(\Omega), \\
& \left(L_{3}(\Omega), W_{3, \mathcal{B}}^{2}(\Omega)\right)_{11 / 24,3} \doteq W_{3, \mathcal{B}}^{11 / 12}(\Omega),
\end{aligned}
$$

by [3, Eq. (5.3) and Theorem 5.2], we infer from (1.1c) and regularizing properties of the semigroup in $L_{3}(\Omega)$ associated with the unbounded operator $-D \Delta+\alpha$ id with domain $W_{3, \mathcal{B}}^{2}(\Omega)$ that, for $t \in\left[0, T_{m}\right)$,

$$
\begin{aligned}
\|w(t)\|_{W_{\infty}^{1}(\Omega)} & \leq C\|w(t)\|_{W_{3}^{11 / 6}(\Omega)} \\
& \leq C e^{-\alpha t / 2}(D t)^{-11 / 24}\left\|w_{0}\right\|_{W_{3}^{11 / 12}(\Omega)}+C \int_{0}^{t} e^{-\alpha(t-s) / 2}(D(t-s))^{-11 / 24}\|v(s)\|_{W_{3}^{11 / 12}(\Omega)} \mathrm{d} s \\
& \leq C t^{-11 / 24}+C\left(\sup _{s \in[0, t]}\left\{\|v(s)\|_{W_{3}^{1}(\Omega)}\right\}\right) t^{13 / 24}
\end{aligned}
$$

where we have used the continuous embedding of $W_{3}^{1}(\Omega)$ in $W_{3}^{11 / 12}(\Omega)$ to obtain the last inequality. Since $v \in C\left(\left[0, T_{m}\right) ; W_{3}^{1}(\Omega)\right)$, the above inequality implies that, for $T \in\left(0, T_{m}\right)$,

$$
\int_{0}^{T}\|w(t)\|_{W_{\infty}^{1}(\Omega)}^{2} \mathrm{~d} t \leq C T^{1 / 12}+C\left(\sup _{s \in[0, t]}\left\{\|v(s)\|_{W_{3}^{1}(\Omega)}\right\}\right)^{2} T^{25 / 12}
$$

that is,

$$
w \in L_{2}\left((0, T) ; W_{\infty}^{1}(\Omega)\right), \quad T \in\left(0, T_{m}\right) .
$$

Let us now recall that the positive part $r_{+}$of a real number is given by $r_{+}:=\max \{r, 0\}$ and set $N(r):=(-r)_{+}$for $r \in \mathbb{R}$. Then,

$$
N^{\prime}(r)^{2}=-N^{\prime}(r), \quad r N^{\prime}(r)=N(r), \quad\left(N N^{\prime}\right)(r)=-N(r), \quad r N(r)=-N(r)^{2} .
$$

On the one hand, it follows from (1.1a), (3.4), and Young's inequality that

$$
\begin{aligned}
\frac{1}{2} \frac{\mathrm{d}}{\mathrm{d} t}\|N(u)\|_{L_{2}(\Omega)}^{2}= & \int_{\Omega} N^{\prime}(u)|\nabla u|^{2} \mathrm{~d} x-\int_{\Omega} u N^{\prime}(u) \nabla u \cdot \nabla w \mathrm{~d} x \\
& -\theta \int_{\Omega} N(u)\left(v_{+}-N(v)\right) \mathrm{d} x-\|N(u)\|_{L_{2}(\Omega)}^{2}
\end{aligned}
$$




$$
\begin{aligned}
\leq- & \|\nabla N(u)\|_{L_{2}(\Omega)}^{2}+\int_{\Omega} u N^{\prime}(u)^{2} \nabla u \cdot \nabla w \mathrm{~d} x \\
& +\theta \int_{\Omega} N(u) N(v) \mathrm{d} x-\|N(u)\|_{L_{2}(\Omega)}^{2} \\
\leq- & \|\nabla N(u)\|_{L_{2}(\Omega)}^{2}+\int_{\Omega} N(u) \nabla N(u) \cdot \nabla w \mathrm{~d} x \\
& +\theta \int_{\Omega} N(u) N(v) \mathrm{d} x-\|N(u)\|_{L_{2}(\Omega)}^{2} \\
\leq- & \frac{1}{2}\|\nabla N(u)\|_{L_{2}(\Omega)}^{2}+\frac{1}{2}\|N(u)\|_{L_{2}(\Omega)}^{2}\|\nabla w\|_{L_{\infty}(\Omega)}^{2} \\
& +\theta \int_{\Omega} N(u) N(v) \mathrm{d} x-\|N(u)\|_{L_{2}(\Omega)}^{2} .
\end{aligned}
$$

On the other hand, we infer from (1.1b) and (3.4) that

$$
\begin{aligned}
\frac{\theta}{2} \frac{\mathrm{d}}{\mathrm{d} t}\|N(v)\|_{L_{2}(\Omega)}^{2} & =-\theta \int_{\Omega} N(v)\left(u_{+}-N(u)-\theta v\right) \mathrm{d} x \\
& \leq \theta \int_{\Omega} N(v) N(u) \mathrm{d} x-\theta^{2}\|N(v)\|_{L_{2}(\Omega}^{2} .
\end{aligned}
$$

Summing up the previous two differential inequalities leads us to

$$
\begin{aligned}
\frac{\mathrm{d}}{\mathrm{d} t}\left(\|N(u)\|_{L_{2}(\Omega)}^{2}+\theta\|N(v)\|_{L_{2}(\Omega)}^{2}\right) & \leq\|N(u)\|_{L_{2}(\Omega)}^{2}\|w\|_{W_{\infty}^{1}(\Omega)}^{2}-2\|N(u)-\theta N(v)\|_{L_{2}(\Omega)}^{2} \\
& \leq\|w\|_{W_{\infty}^{1}(\Omega)}^{2}\left(\|N(u)\|_{L_{2}(\Omega)}^{2}+\theta\|N(v)\|_{L_{2}(\Omega)}^{2}\right) .
\end{aligned}
$$

Hence, by (3.3) and the nonnegativity of $u_{0}$ and $v_{0}$,

$$
\begin{aligned}
& \|N(u(t))\|_{L_{2}(\Omega)}^{2}+\theta\|N(v(t))\|_{L_{2}(\Omega)}^{2} \\
& \quad \leq\left(\left\|N\left(u_{0}\right)\right\|_{L_{2}(\Omega)}^{2}+\theta\left\|N\left(v_{0}\right)\right\|_{L_{2}(\Omega)}^{2}\right) \exp \left\{\int_{0}^{t}\|w(s)\|_{W_{\infty}^{1}(\Omega)}^{2} \mathrm{~d} s\right\}=0
\end{aligned}
$$

for $t \in\left[0, T_{m}\right)$, from which the nonnegativity of $u$ and $v$ in $\left[0, T_{m}\right)$ follows. That $w$ is also nonnegative in $\left[0, T_{m}\right)$ is next a straightforward consequence of that of $v$ and the comparison principle applied to (1.1c).

We finally integrate (1.1a) and (1.1b) over $\Omega$ and deduce from the no-flux boundary conditions (1.1d) that

$$
\frac{\mathrm{d}}{\mathrm{d} t} \int_{\Omega}(u+v) \mathrm{d} x=\int_{\Omega} \operatorname{div}(\nabla u-u \nabla w) \mathrm{d} x=0, \quad t \in\left[0, T_{m}\right),
$$

from which (3.1) follows by the nonnegativity of $u$ and $v$. 
3.2. Smoothness for positive times. Owing to the regularizing properties of the Laplace operator and the associated semigroup in $L_{3}(\Omega)$, the solution to (1.1)-(1.2) constructed in Proposition 3.1 is more regular for positive times, as reported in the next result.

Corollary 3.2. Let the assumptions from Proposition 3.1 be fulfilled and denote the weak solution to (1.1)-(1.2) constructed in Proposition 3.1 by $(u, v, w)$. Then

$$
\begin{aligned}
& u \in C\left(\left(0, T_{m}\right) ; W_{3, \mathcal{B}}^{2}(\Omega)\right) \cap C^{1}\left(\left(0, T_{m}\right) ; L_{3}(\Omega)\right), \\
& w \in C\left(\left(0, T_{m}\right) ; W_{3, \mathcal{B}}^{2}(\Omega)\right) \cap C^{1}\left(\left(0, T_{m}\right) ; L_{3}(\Omega)\right) .
\end{aligned}
$$

Proof. We adapt and refine the proof of $\left[17\right.$, Corollary 2.2]. Let $\beta \in(0,1)$. Since $v \in C^{1}\left(\left[0, T_{m}\right) ; W_{3}^{1}(\Omega)\right)$ and $-D \Delta+\alpha$ id generates an analytic semigroup in $L_{3}(\Omega)$, we infer from (1.1c) and [3, Theorem 10.1] (with $\rho=\beta, E_{0}=L_{3}(\Omega)$, and $E_{1}=W_{3, \mathcal{B}}^{2}(\Omega)$ ) that

$$
w \in C^{\beta}\left(\left(0, T_{m}\right) ; W_{3, \mathcal{B}}^{2}(\Omega)\right) \cap C^{1+\beta}\left(\left(0, T_{m}\right) ; L_{3}(\Omega)\right) .
$$

Next, fix $\gamma \in(5 / 6,1)$. On the one hand, by (1.3) and Proposition 3.1,

$$
u \in C\left(\left[0, T_{m}\right) ; W_{3, \mathcal{B}}^{1}(\Omega)\right) \cap C^{1}\left(\left[0, T_{m}\right) ; W_{3, \mathcal{B}}^{-1}(\Omega)\right),
$$

while [3, Theorem 7.2] guarantees that

$$
\left(W_{3, \mathcal{B}}^{-1}(\Omega), W_{3, \mathcal{B}}^{1}(\Omega)\right)_{\gamma, 3} \doteq W_{3, \mathcal{B}}^{2 \gamma-1}(\Omega)
$$

(up to equivalent norms). Then, for $0 \leq s \leq t<T_{m}$,

$$
\begin{aligned}
\|u(t)-u(s)\|_{W_{3, \mathcal{B}}^{2 \gamma-1}(\Omega)} & \leq\|u(t)-u(s)\|_{W_{3, \mathcal{B}}^{-1}(\Omega)}^{1-\gamma}\|u(t)-u(s)\|_{W_{3, \mathcal{B}}^{1}(\Omega)}^{\gamma} \\
& \leq 2^{\gamma}(t-s)^{1-\gamma} \sup _{[s, t]}\left\|\partial_{t} u\right\|_{W_{3, \mathcal{B}}^{-1}(\Omega)}^{1-\gamma} \sup _{[s, t]}\|u\|_{W_{3, \mathcal{B}}^{1}(\Omega)}^{\gamma},
\end{aligned}
$$

and we deduce from (3.8) that

$$
u \in C^{1-\gamma}\left(\left[0, T_{m}\right) ; W_{3, \mathcal{B}}^{2 \gamma-1}(\Omega)\right) .
$$

On the other hand, using (3.7) with $\beta=1-\gamma$ along with the continuous embedding of $W_{3, \mathcal{B}}^{2}(\Omega)$ in $W_{3, \mathcal{B}}^{2 \gamma}(\Omega)$, we obtain that

$$
w \in C^{1-\gamma}\left(\left(0, T_{m}\right) ; W_{3, \mathcal{B}}^{2 \gamma}(\Omega)\right) .
$$

Owing to the choice of $\gamma$ which ensures that $2 \gamma-1>2 / 3$, the space $W_{3, \mathcal{B}}^{2 \gamma-1}(\Omega)$ is an algebra and we deduce from (3.9) and (3.10) that $u \nabla w \in C^{1-\gamma}\left(\left(0, T_{m}\right) ; W_{3, \mathcal{B}}^{2 \gamma-1}\left(\Omega ; \mathbb{R}^{2}\right)\right)$. Thus,

$$
\operatorname{div}(u \nabla w) \in C^{1-\gamma}\left(\left(0, T_{m}\right) ; W_{3, \mathcal{B}}^{2 \gamma-2}(\Omega)\right) .
$$

Now, let $\varepsilon \in\left(0, T_{m}\right)$. Thanks to [3, Theorem 8.5], the realization in $W_{3, \mathcal{B}}^{2 \gamma-2}(\Omega)$ of the Laplace operator with homogeneous Neumann boundary conditions generates an analytic semigroup in $W_{3, \mathcal{B}}^{2 \gamma-2}(\Omega)$, its domain being $W_{3, \mathcal{B}}^{2 \gamma}(\Omega)$, and it follows from (1.1a), (3.11), and [3, Theorem 10.1] (with $\rho=1-\gamma$, $E_{0}=W_{3, \mathcal{B}}^{2 \gamma-2}(\Omega)$, and $\left.E_{1}=W_{3, \mathcal{B}}^{2 \gamma}(\Omega)\right)$ that

$$
u \in C^{1-\gamma}\left(\left(\varepsilon / 2, T_{m}\right) ; W_{3, \mathcal{B}}^{2 \gamma}(\Omega)\right) \cap C^{2-\gamma}\left(\left(\varepsilon / 2, T_{m}\right) ; W_{3, \mathcal{B}}^{2 \gamma-2}(\Omega)\right) .
$$


Finally, fix $\eta \in(5 / 3-\gamma, 1)$ and notice that [3, Theorem 7.2] guarantees that, up to equivalent norms,

$$
\left(W_{3, \mathcal{B}}^{2 \gamma-2}(\Omega), W_{3, \mathcal{B}}^{2 \gamma}(\Omega)\right)_{\eta, 3} \doteq W_{3, \mathcal{B}}^{2(\eta+\gamma)-2}(\Omega) .
$$

Therefore, for $\varepsilon / 2<s \leq t<T_{m}$,

$$
\begin{aligned}
\|u(t)-u(s)\|_{W_{3, \mathcal{B}}^{2(\eta+\gamma)-2}(\Omega)} & \leq\|u(t)-u(s)\|_{W_{3, \mathcal{B}}^{2 \gamma-2}(\Omega)}^{1-\eta}\|u(t)-u(s)\|_{W_{3, \mathcal{B}}^{2 \gamma}(\Omega)}^{\eta} \\
& \leq(t-s)^{1-\eta} \sup _{[s, t]}\left\|\partial_{t} u\right\|_{W_{3, \mathcal{B}}^{2 \gamma-2}(\Omega)}^{1-\eta}(t-s)^{\eta(1-\gamma)}\|u\|_{C^{1-\gamma\left([s, t] ; W_{3, \mathcal{B}}^{1}(\Omega)\right)}}^{\eta},
\end{aligned}
$$

and we infer from (3.12), that

$$
u \in C^{1-\gamma \eta}\left(\left(\varepsilon / 2, T_{m}\right) ; W_{3, \mathcal{B}}^{2(\eta+\gamma)-2}(\Omega)\right) .
$$

Hence, since $2(\eta+\gamma)-2>4 / 3>1$,

$$
u \in C^{1-\gamma \eta}\left(\left(\varepsilon / 2, T_{m}\right) ; W_{3, \mathcal{B}}^{1}(\Omega)\right) .
$$

Combining (3.7) (with $\beta=1-\gamma \eta$ ) and (3.13) and recalling that $W_{3, \mathcal{B}}^{1}(\Omega)$ is an algebra entail that $u \nabla w$ belongs to $C^{1-\gamma \eta}\left(\left(\varepsilon / 2, T_{m}\right) ; W_{3, \mathcal{B}}^{1}\left(\Omega ; \mathbb{R}^{2}\right)\right)$ and thus

$$
\operatorname{div}(u \nabla w) \in C^{1-\gamma \eta}\left(\left(\varepsilon / 2, T_{m}\right) ; L_{3}(\Omega)\right) .
$$

In view of (1.1a) and (3.14), another application of [3, Theorem 10.1] (with $\rho=1-\gamma \eta, E_{0}=L_{3}(\Omega)$, $\left.E_{1}=W_{3, \mathcal{B}}^{2}(\Omega)\right)$ gives

$$
u \in C^{1-\gamma \eta}\left(\left(\varepsilon, T_{m}\right) ; W_{3, \mathcal{B}}^{2}(\Omega)\right) \cap C^{2-\gamma \eta}\left(\left(\varepsilon, T_{m}\right) ; L_{3}(\Omega)\right) .
$$

Since $\varepsilon \in\left(0, T_{m}\right)$ is arbitrary, the proof of Corollary 3.2 is complete.

3.3. Estimates and global existence. We now prove the global existence for (1.1)-(1.2) and aim at showing that the solution $(u, v, w)$ from Proposition 3.1 satisfies (3.2) for all $T>0$. To this end, we take advantage of the outcome of Corollary 3.2 which guarantees higher regularity for $(u(t), v(t), w(t))$ for $t \in\left(0, T_{m}\right)$ and derive estimates on $\left[t_{0}, T_{m}\right)$ for some fixed but arbitrary $t_{0} \in\left(0, T_{m}\right)$.

Let us thus fix $t_{0} \in\left(0, T_{m}\right)$ and recall that Corollary 3.2 ensures that

$$
\left(u\left(t_{0}\right), w\left(t_{0}\right)\right) \in W_{3, \mathcal{B}}^{2}\left(\Omega ; \mathbb{R}^{2}\right), \quad v\left(t_{0}\right) \in W_{3}^{1}(\Omega), \quad \text { and } \quad \partial_{t} w\left(t_{0}\right) \in L_{3}(\Omega) .
$$

For further use, we also fix

$$
\gamma \in(5 / 6,1)
$$

see Corollary 3.9 and Lemma 3.10. For the remainder of this section, $C$ and $\left(C_{i}\right)_{i \geq 1}$ denote positive constants depending only on $\Omega, \theta, D, \alpha, \gamma, t_{0},\left\|u\left(t_{0}\right)\right\|_{W_{3}^{2}(\Omega)},\left\|v\left(t_{0}\right)\right\|_{W_{3}^{1}(\Omega)}$, and $\left\|w\left(t_{0}\right)\right\|_{W_{3}^{2}(\Omega)}$. Dependence upon additional parameters is indicated explicitly. We first remark that the mass conservation (3.1), in conjunction with the nonnegativity of $u$ and $v$, implies

$$
\|u(t)\|_{L_{1}(\Omega)} \leq M \quad \text { and } \quad\|v(t)\|_{L_{1}(\Omega)} \leq M, \quad t \in\left[t_{0}, T_{m}\right)
$$

We next derive a series of estimates. 
Lemma 3.3. Let $T>t_{0}$. There is $C_{1}(T)>0$ such that, for $t \in\left[t_{0}, T\right] \cap\left[t_{0}, T_{m}\right)$,

$$
\begin{aligned}
\|L(u(t))\|_{L_{1}(\Omega)}+ & \|v(t)\|_{L_{2}(\Omega)}+\|w(t)\|_{W_{2}^{1}(\Omega)}+\left\|\partial_{t} w(t)\right\|_{L_{2}(\Omega)} \leq C_{1}(T), \\
& \int_{t_{0}}^{t}\left(\|\nabla \sqrt{u}(s)\|_{L_{2}(\Omega)}^{2}+\left\|\partial_{t} w(s)\right\|_{W_{2}^{1}(\Omega)}^{2}\right) \mathrm{d} s \leq C_{1}(T),
\end{aligned}
$$

where $L$ is defined in (2.1).

Proof. Using (1.1a), (1.1b), (1.1d) along with integration by parts, we obtain

$$
\begin{aligned}
\frac{\mathrm{d}}{\mathrm{d} t}\left(\|L(u)\|_{L_{1}(\Omega)}+\left\|L_{\theta}(v)\right\|_{L_{1}(\Omega)}\right) & \\
& =-\int_{\Omega} \frac{|\nabla u|^{2}}{u} \mathrm{~d} x+\int_{\Omega} \nabla u \cdot \nabla w \mathrm{~d} x+\int_{\Omega}(\ln u-\ln (\theta v))(\theta v-u) \mathrm{d} x \\
& =-4\|\nabla \sqrt{u}\|_{L_{2}(\Omega)}^{2}-\int_{\Omega} u \Delta w \mathrm{~d} x-\int_{\Omega}(\ln (\theta v)-\ln u)(\theta v-u) \mathrm{d} x \\
& \leq-4\|\nabla \sqrt{u}\|_{L_{2}(\Omega)}^{2}-\int_{\Omega} u \Delta w \mathrm{~d} x,
\end{aligned}
$$

in view of the monotonicity of the logarithm. Due to $(1.1 \mathrm{~b})-(1.1 \mathrm{~d})$, we further have

$$
\begin{aligned}
\int_{\Omega} u \partial_{t} w \mathrm{~d} x= & \int_{\Omega}\left(\partial_{t} v \partial_{t} w+\theta v \partial_{t} w\right) \mathrm{d} x \\
= & \int_{\Omega} \partial_{t} w\left(\partial_{t}^{2} w-D \Delta \partial_{t} w+\alpha \partial_{t} w\right) \mathrm{d} x+\theta \int_{\Omega} \partial_{t} w\left(\partial_{t} w-D \Delta w+\alpha w\right) \mathrm{d} x \\
= & \frac{1}{2} \frac{\mathrm{d}}{\mathrm{d} t}\left(\left\|\partial_{t} w\right\|_{L_{2}(\Omega)}^{2}+\theta D\|\nabla w\|_{L_{2}(\Omega)}^{2}+\theta \alpha\|w\|_{L_{2}(\Omega)}^{2}\right) \\
& +D\left\|\nabla \partial_{t} w\right\|_{L_{2}(\Omega)}^{2}+(\alpha+\theta)\left\|\partial_{t} w\right\|_{L_{2}(\Omega)}^{2} .
\end{aligned}
$$

Introducing

$$
Y:=D\left(\|L(u)\|_{L_{1}(\Omega)}+\left\|L_{\theta}(v)\right\|_{L_{1}(\Omega)}\right)+\frac{1}{2}\left(\left\|\partial_{t} w\right\|_{L_{2}(\Omega)}^{2}+\theta D\|\nabla w\|_{L_{2}(\Omega)}^{2}+\theta \alpha\|w\|_{L_{2}(\Omega)}^{2}\right),
$$

we combine (3.18) and (3.19) and deduce from (1.1c) that

$$
\begin{aligned}
\frac{\mathrm{dY}}{\mathrm{d} t}+4 D\|\nabla \sqrt{u}\|_{L_{2}(\Omega)}^{2} & +D\left\|\nabla \partial_{t} w\right\|_{L_{2}(\Omega)}^{2}+(\alpha+\theta)\left\|\partial_{t} w\right\|_{L_{2}(\Omega)}^{2} \\
& \leq \int_{\Omega} u\left(\partial_{t} w-D \Delta w\right) \mathrm{d} x=\int_{\Omega} u(v-\alpha w) \mathrm{d} x .
\end{aligned}
$$

Also, by (1.1b),

$$
\frac{1}{2} \frac{\mathrm{d}}{\mathrm{d} t}\|v\|_{L_{2}(\Omega)}^{2}=\int_{\Omega} u v \mathrm{~d} x-\theta\|v\|_{L_{2}(\Omega)}^{2} \leq \int_{\Omega} u v \mathrm{~d} x
$$


Summing the previous two inequalities and using the nonnegativity of $u$ and $w$, we find

$$
\begin{aligned}
\frac{\mathrm{d}}{\mathrm{d} t}\left(Y+\frac{1}{2}\|v\|_{L_{2}(\Omega)}^{2}\right)+4 D\|\nabla \sqrt{u}\|_{L_{2}(\Omega)}^{2} & +D\left\|\nabla \partial_{t} w\right\|_{L_{2}(\Omega)}^{2}+(\alpha+\theta)\left\|\partial_{t} w\right\|_{L_{2}(\Omega)}^{2} \\
& \leq 2 \int_{\Omega} u v \mathrm{~d} x .
\end{aligned}
$$

Since $\Omega \subset \mathbb{R}^{2}$, we infer from (3.17) and Hölder's, Gagliardo-Nirenberg's and Young's inequalities that

$$
\begin{aligned}
2 \int_{\Omega} u v \mathrm{~d} x & \leq 2\|u\|_{L_{2}(\Omega)}\|v\|_{L_{2}(\Omega)}=2\|v\|_{L_{2}(\Omega)}\|\sqrt{u}\|_{L_{4}(\Omega)}^{2} \\
& \leq C\|v\|_{L_{2}(\Omega)}\left(\|\nabla \sqrt{u}\|_{L_{2}(\Omega)}\|\sqrt{u}\|_{L_{2}(\Omega)}+\|\sqrt{u}\|_{L_{2}(\Omega)}^{2}\right) \\
& \leq C\|v\|_{L_{2}(\Omega)}\left(\sqrt{M}\|\nabla \sqrt{u}\|_{L_{2}(\Omega)}+M\right) \\
& \leq 2 D\|\nabla \sqrt{u}\|_{L_{2}(\Omega)}^{2}+C\left(1+\|v\|_{L_{2}(\Omega)}^{2}\right) .
\end{aligned}
$$

Combining (3.20) and (3.21) and rearranging the terms, we conclude that

$$
\begin{aligned}
\frac{\mathrm{d}}{\mathrm{d} t}\left(Y+\frac{1}{2}\|v\|_{L_{2}(\Omega)}^{2}\right)+2 D\|\nabla \sqrt{u}\|_{L_{2}(\Omega)}^{2} & +D\left\|\nabla \partial_{t} w\right\|_{L_{2}(\Omega)}^{2}+(\alpha+\theta)\left\|\partial_{t} w\right\|_{L_{2}(\Omega)}^{2} \\
& \leq C\left(1+\|v\|_{L_{2}(\Omega)}^{2}\right) .
\end{aligned}
$$

Applying first Gronwall's inequality and then the time integrated version of the previous inequality, we deduce the claim in view of the positivity of all the terms involved in the left hand side of the above inequality and the finiteness of $Y\left(t_{0}\right)$ stemming from (3.15).

Corollary 3.4. Let $T>t_{0}$. There is $C_{2}(T)>0$ such that

$$
\|w(t)\|_{W_{2}^{2}(\Omega)} \leq C_{2}(T), \quad t \in\left[t_{0}, T\right] \cap\left[t_{0}, T_{m}\right) .
$$

Proof. Using (1.1c) along with Lemma 3.3, we obtain, for $t \in\left[t_{0}, T\right] \cap\left[t_{0}, T_{m}\right)$,

$$
D\|\Delta w(t)\|_{L_{2}(\Omega)} \leq\left\|\partial_{t} w(t)\right\|_{L_{2}(\Omega)}+\alpha\|w(t)\|_{L_{2}(\Omega)}+\|v(t)\|_{L_{2}(\Omega)} \leq(2+\alpha) C_{1}(T) .
$$

Since $w(t) \in W_{3, \mathcal{B}}^{2}(\Omega)$ for $t \in\left[t_{0}, T\right] \cap\left[t_{0}, T_{m}\right)$ by Corollary 3.2, the claim follows from CalderonZygmund's estimate which guarantees that $\|z\|_{W_{2}^{2}(\Omega)} \leq C\left(\|\Delta z\|_{L_{2}(\Omega)}+\|z\|_{L_{2}(\Omega)}\right)$ for all $z \in W_{2, \mathcal{B}}^{2}(\Omega)$.

Lemma 3.5. Let $T>t_{0}$ and $r>0$. There is $C_{3}(T, r)>0$ such that

$$
\|u(t)\|_{L_{r+1}(\Omega)}+\|v(t)\|_{L_{r+1}(\Omega)} \leq C_{3}(T, r), \quad t \in\left[t_{0}, T\right] \cap\left[t_{0}, T_{m}\right) .
$$

Proof. Let $r>0$. Using (1.1) and integration by parts gives

$$
\frac{\mathrm{d}}{\mathrm{d} t}\left(\|u\|_{L_{r+1}(\Omega)}^{r+1}+\theta^{r}\|v\|_{L_{r+1}(\Omega)}^{r+1}\right)=-r(r+1) \int_{\Omega} u^{r-1}|\nabla u|^{2} \mathrm{~d} x+r(r+1) \int_{\Omega} u^{r} \nabla u \cdot \nabla w \mathrm{~d} x
$$




$$
\begin{gathered}
+(r+1) \int_{\Omega}(u-\theta v)\left(\theta^{r} v^{r}-u^{r}\right) \mathrm{d} x \\
\leq-\frac{4 r}{r+1}\left\|\nabla\left(u^{(r+1) / 2}\right)\right\|_{L_{2}(\Omega)}^{2}-r \int_{\Omega} u^{r+1} \Delta w \mathrm{~d} x .
\end{gathered}
$$

It now follows from Hölder's and Gagliardo-Nirenberg's inequalities that

$$
\begin{aligned}
r\left|\int_{\Omega} u^{r+1} \Delta w \mathrm{~d} x\right| & \leq r\|\Delta w\|_{L_{2}(\Omega)}\left\|u^{(r+1) / 2}\right\|_{L_{4}(\Omega)}^{2} \\
& \leq r C\|\Delta w\|_{L_{2}(\Omega)}\left(\left\|\nabla u^{(r+1) / 2}\right\|_{L_{2}(\Omega)}\left\|u^{(r+1) / 2}\right\|_{L_{2}(\Omega)}+\left\|u^{(r+1) / 2}\right\|_{L_{2}(\Omega)}^{2}\right) \\
& \leq \frac{2 r}{r+1}\left\|\nabla u^{(r+1) / 2}\right\|_{L_{2}(\Omega)}^{2}+C r(r+1)\|\Delta w\|_{L_{2}(\Omega)}^{2}\left\|u^{(r+1) / 2}\right\|_{L_{2}(\Omega)}^{2} \\
& +C r\|\Delta w\|_{L_{2}(\Omega)}\left\|u^{(r+1) / 2}\right\|_{L_{2}(\Omega)}^{2} \\
& \leq \frac{2 r}{r+1}\left\|\nabla u^{(r+1) / 2}\right\|_{L_{2}(\Omega)}^{2}+C r(r+1)\left(1+\|\Delta w\|_{L_{2}(\Omega)}^{2}\right)\|u\|_{L_{r+1}(\Omega)}^{r+1} .
\end{aligned}
$$

Combining the above two inequalities with Corollary 3.4, we obtain

$$
\begin{aligned}
\frac{\mathrm{d}}{\mathrm{d} t}\left(\|u\|_{L_{r+1}(\Omega)}^{r+1}+\theta^{r}\|v\|_{L_{r+1}(\Omega)}^{r+1}\right) \leq & -\frac{2 r}{r+1}\left\|\nabla u^{(r+1) / 2}\right\|_{L_{2}(\Omega)}^{2} \\
& +C r(r+1)\left(1+C_{2}(T)^{2}\right)\|u\|_{L_{r+1}(\Omega)}^{r+1} .
\end{aligned}
$$

Then Gronwall's inequality implies the claim.

Lemma 3.6. Let $T>t_{0}$. There is $C_{4}(T)>0$ such that

$$
\|u(t)\|_{L_{\infty}(\Omega)}+\|v(t)\|_{L_{\infty}(\Omega)}+\left\|\partial_{t} v(t)\right\|_{L_{\infty}(\Omega)} \leq C_{4}(T), \quad t \in\left[t_{0}, T\right] \cap\left[t_{0}, T_{m}\right) .
$$

Proof. Let $T>t_{0}$ and $I:=\left[t_{0}, T\right] \cap\left[t_{0}, T_{m}\right)$. In view of (1.1a) and (1.1d), $u$ satisfies

$$
\partial_{t} u=\operatorname{div}(D \nabla u)+\operatorname{div} \mathbf{f}+g, \quad(t, x) \in\left(t_{0}, T_{m}\right) \times \Omega,
$$

along with no-flux boundary conditions, where $D \equiv 1, \mathbf{f}:=-u \nabla w$, and $g:=\theta v-u$. According to Lemma 3.5,

$$
u \in L_{\infty}\left(I ; L_{1}(\Omega)\right) \text { and } g \in L_{\infty}\left(I ; L_{3}(\Omega)\right)
$$

while Corollary 3.4, Lemma 3.5, and the continuous embedding of $W_{2}^{2}(\Omega)$ in $W_{r}^{1}(\Omega)$ for any $r \in[1, \infty)$ ensure that $\mathbf{f} \in L_{\infty}\left(I ; L_{5}\left(\Omega ; \mathbb{R}^{2}\right)\right)$. We then infer from [33, Lemma A.1] that

$$
\|u(t)\|_{L_{\infty}(\Omega)} \leq C(T), \quad t \in\left[t_{0}, T\right] \cap\left[t_{0}, T_{m}\right) .
$$

Combining this estimate with $(1.1 \mathrm{~b}),(3.15)$, the nonnegativity of $v$, and the continuous embedding of $W_{3}^{1}(\Omega)$ in $L_{\infty}(\Omega)$, we further obtain

$$
0 \leq v(t, x)=e^{-\theta\left(t-t_{0}\right)} v\left(t_{0}, x\right)+\int_{t_{0}}^{t} u(s, x) e^{-\theta(t-s)} \mathrm{d} s
$$




$$
\begin{aligned}
& \leq e^{-\theta\left(t-t_{0}\right)}\left\|v\left(t_{0}\right)\right\|_{L_{\infty}(\Omega)}+\int_{t_{0}}^{t}\|u(s)\|_{L_{\infty}(\Omega)} e^{-\theta(t-s)} \mathrm{d} s \\
& \leq C\left\|v\left(t_{0}\right)\right\|_{W_{3}^{1}(\Omega)}+\frac{C(T)}{\theta} \leq C(T)
\end{aligned}
$$

for $(t, x) \in\left[t_{0}, T\right] \cap\left[t_{0}, T_{m}\right)$. Finally, the last part of the claim immediately follows from (1.1b).

Corollary 3.7. Let $T>t_{0}$. There is $C_{5}(T)>0$ such that

$$
\|w(t)\|_{W_{\infty}^{1}(\Omega)} \leq C_{5}(T), \quad t \in\left[t_{0}, T\right] \cap\left[t_{0}, T_{m}\right) .
$$

Proof. The proof is similar to that of [17, Corollary 2.7] and we recall it here for the sake of completeness. Owing to the continuous embedding of $W_{3}^{11 / 6}(\Omega)$ in $W_{\infty}^{1}(\Omega)$, see [25, Theorem 7.1.2], and

$$
\left(L_{3}(\Omega), W_{3, \mathcal{B}}^{2}(\Omega)\right)_{11 / 12,3} \doteq W_{3, \mathcal{B}}^{11 / 6}(\Omega)
$$

see [3, Theorem 7.2], it follows from (1.1c), (3.15), Duhamel's formula, the regularizing properties of the heat semigroup, see [4, Theorem V.2.1.3], and Lemma 3.6 that, for $t \in\left[t_{0}, T\right] \cap\left[t_{0}, T_{m}\right)$,

$$
\begin{aligned}
\|w(t)\|_{W_{\infty}^{1}(\Omega)} & \leq C\|w(t)\|_{W_{3}^{11 / 6}(\Omega)} \\
& \leq C e^{-\alpha\left(t-t_{0}\right) / 2}\left\|w\left(t_{0}\right)\right\|_{W_{3}^{11 / 6}(\Omega)}+C \int_{t_{0}}^{t} e^{-\alpha(t-s) / 2}(t-s)^{-11 / 12}\|v(s)\|_{L_{3}(\Omega)} \mathrm{d} s \\
& \leq C\left\|w\left(t_{0}\right)\right\|_{W_{3}^{2}(\Omega)}+C C_{4}(T) \int_{t_{0}}^{t}(t-s)^{-11 / 12} \mathrm{~d} s \leq C(T),
\end{aligned}
$$

and the proof is complete.

Lemma 3.8. Let $T>t_{0}$. There is $C_{6}(T)>0$ such that

$$
\|\nabla u(t)\|_{L_{2}(\Omega)}+\int_{t_{0}}^{t}\left\|\partial_{t} u(s)\right\|_{L_{2}(\Omega)}^{2} \mathrm{~d} s \leq C_{6}(T), \quad t \in\left[t_{0}, T\right] \cap\left[t_{0}, T_{m}\right) .
$$

Proof. Multiplying (1.1a) by $\partial_{t} u$ and integrating over $\Omega$, we conclude from Hölder's and Young's inequalities that

$$
\begin{aligned}
\left\|\partial_{t} u\right\|_{L_{2}(\Omega)}^{2}+ & \frac{1}{2} \frac{\mathrm{d}}{\mathrm{d} t}\|u\|_{W_{2}^{1}(\Omega)}^{2}=-\int_{\Omega} \partial_{t} u(u \Delta w+\nabla u \cdot \nabla w) \mathrm{d} x+\theta \int_{\Omega} v \partial_{t} u \mathrm{~d} x \\
\leq & \left\|\partial_{t} u\right\|_{L_{2}(\Omega)}\|u\|_{L_{\infty}(\Omega)}\|\Delta w\|_{L_{2}(\Omega)}+\left\|\partial_{t} u\right\|_{L_{2}(\Omega)}\|\nabla u\|_{L_{2}(\Omega)}\|\nabla w\|_{L_{\infty}(\Omega)} \\
& \quad+\theta\left\|\partial_{t} u\right\|_{L_{2}(\Omega)}\|v\|_{L_{2}(\Omega)} \\
\leq & \frac{1}{2}\left\|\partial_{t} u\right\|_{L_{2}(\Omega)}^{2}+\frac{3}{2}\left(\|u\|_{L_{\infty}(\Omega)}^{2}\|\Delta w\|_{L_{2}(\Omega)}^{2}+\|\nabla u\|_{L_{2}(\Omega)}^{2}\|\nabla w\|_{L_{\infty}(\Omega)}^{2}+\theta^{2}\|v\|_{L_{2}(\Omega)}^{2}\right) .
\end{aligned}
$$

Lemmas 3.3 and 3.6 and Corollaries 3.4 and 3.7 next imply that

$$
\left\|\partial_{t} u\right\|_{L_{2}(\Omega)}^{2}+\frac{\mathrm{d}}{\mathrm{d} t}\|u\|_{W_{2}^{1}(\Omega)}^{2} \leq C(T)\left(1+\|\nabla u\|_{L_{2}(\Omega)}^{2}\right),
$$

so that Gronwall's inequality yields the claim. 
Corollary 3.9. Let $T>t_{0}$. There is $C_{7}(T)>0$ such that

$$
\|u(t)\|_{W_{3}^{1}(\Omega)}+\|u(t)\|_{W_{2}^{2 \gamma}(\Omega)}+\|v(t)\|_{W_{3}^{1}(\Omega)} \leq C_{7}(T), \quad t \in\left[t_{0}, T\right] \cap\left[t_{0}, T_{m}\right),
$$

the parameter $\gamma \in(5 / 6,1)$ being defined in (3.16).

Proof. Using (1.1a) along with properties of the heat semigroup (see, e.g., [1, Proposition 12.5] and [4, Theorem V.2.1.3]) and Hölder's inequality, we deduce from (3.15), Corollaries 3.4 and 3.7 as well as Lemmas 3.5, 3.6 and 3.8 that, for $t \in\left[t_{0}, T\right] \cap\left[t_{0}, T_{m}\right)$,

$$
\begin{aligned}
\|\nabla u(t)\|_{L_{3}(\Omega)} \leq & C\left\|\nabla u\left(t_{0}\right)\right\|_{L_{3}(\Omega)}+C \int_{t_{0}}^{t}(t-s)^{-2 / 3}\|(u \Delta w+\nabla u \cdot \nabla w-\theta v+u)(s)\|_{L_{2}(\Omega)} \mathrm{d} s \\
\leq & +C \int_{t_{0}}^{t}(t-s)^{-2 / 3}\left(\|u(s)\|_{L_{\infty}(\Omega)}\|\Delta w(s)\|_{L_{2}(\Omega)}+\|u(s)\|_{L_{2}(\Omega)}\right) \mathrm{d} s \\
& \quad+C \int_{t_{0}}^{t}(t-s)^{-2 / 3}\left(\|\nabla u(s)\|_{L_{2}(\Omega)}\|\nabla w(s)\|_{L_{\infty}(\Omega)}+\theta\|v(s)\|_{L_{2}(\Omega)}\right) \mathrm{d} s \\
\leq C & +C(T) \int_{t_{0}}^{t}(t-s)^{-2 / 3} \mathrm{~d} s \leq C(T) .
\end{aligned}
$$

Combining this estimate with (3.15) and (3.22), we obtain

$$
\|\nabla v(t)\|_{L_{3}(\Omega)} \leq e^{-\theta\left(t-t_{0}\right)}\left\|\nabla v\left(t_{0}\right)\right\|_{L_{3}(\Omega)}+\int_{t_{0}}^{t}\|\nabla u(s)\|_{L_{3}(\Omega)} e^{-\theta(t-s)} \mathrm{d} s \leq C(T) .
$$

Together with Lemma 3.5, the above two estimates entail the stated $W_{3}^{1}$-bounds on $u$ and $v$.

We next invoke (1.1a) along with properties of the heat semigroup (see [4, Theorem V.2.1.3]) and Hölder's inequality to deduce from Corollaries 3.4 and 3.7 and Lemmas 3.5, 3.6 and 3.8 that, for all $t \in\left[t_{0}, T\right] \cap\left[t_{0}, T_{m}\right)$,

$$
\begin{aligned}
\|u(t)\|_{W_{2}^{2 \gamma}(\Omega)} \leq & C\left\|u\left(t_{0}\right)\right\|_{W_{2}^{2 \gamma}(\Omega)}+C \int_{t_{0}}^{t}(t-s)^{-\gamma}\|(u \Delta w+\nabla u \cdot \nabla w-\theta v+u)(s)\|_{L_{2}(\Omega)} \mathrm{d} s \\
\leq & C+C \int_{t_{0}}^{t}(t-s)^{-\gamma}\|u(s)\|_{L_{\infty}(\Omega)}\|\Delta w(s)\|_{L_{2}(\Omega)} \mathrm{d} s \\
& +\int_{t_{0}}^{t}(t-s)^{-\gamma}\|\nabla u(s)\|_{L_{2}(\Omega)}\|\nabla w(s)\|_{L_{\infty}(\Omega)} \mathrm{d} s \\
& +\int_{t_{0}}^{t}(t-s)^{-\gamma}\left(\theta\|v(s)\|_{L_{2}(\Omega)}+\|u(s)\|_{L_{2}(\Omega)}\right) \mathrm{d} s \\
\leq & C+C(T) \int_{t_{0}}^{t}(t-s)^{-\gamma} \mathrm{d} s \leq C(T),
\end{aligned}
$$

and the proof is complete. 
In view of Corollaries 3.7 and 3.9 there is $C_{8}(T)>0$ such that

$$
\|u(t)\|_{W_{3}^{1}(\Omega)}+\|v(t)\|_{W_{3}^{1}(\Omega)}+\|w(t)\|_{W_{3}^{1}(\Omega)} \leq C_{8}(T), \quad t \in\left[t_{0}, T\right] \cap\left[t_{0}, T_{m}\right) .
$$

Hence, according to (3.2), we shall prove Hölder estimates with respect to time in order to conclude the global existence of $(u, v, w)$.

Lemma 3.10. Let $T>t_{0}$ and $\delta:=(3 \gamma-2) / 6 \gamma$, recalling that $\gamma \in(5 / 6,1)$ is defined in (3.16). There is $C_{9}(T)>0$ such that

$$
\left\|u\left(t_{2}\right)-u\left(t_{1}\right)\right\|_{W_{3}^{1}(\Omega)}+\left\|v\left(t_{2}\right)-v\left(t_{1}\right)\right\|_{W_{3}^{1}(\Omega)}+\left\|w\left(t_{2}\right)-w\left(t_{1}\right)\right\|_{W_{3}^{1}(\Omega)} \leq C_{9}(T)\left|t_{2}-t_{1}\right|^{\delta}
$$

for $t_{1}, t_{2} \in\left[t_{0}, T\right] \cap\left[t_{0}, T_{m}\right)$.

Proof. The proof is similar to [17, Lemma 2.10], but is recalled here for the sake of completeness. Let $T>0$ and $t_{1}, t_{2} \in\left[t_{0}, T\right] \cap\left[t_{0}, T_{m}\right)$ such that $t_{2}>t_{1}$.

First, by (1.1b) and (3.23) we have

$$
\left\|\partial_{t} v(t)\right\|_{W_{3}^{1}(\Omega)} \leq\|u(t)\|_{W_{3}^{1}(\Omega)}+\theta\|v(t)\|_{W_{3}^{1}(\Omega)} \leq(1+\theta) C_{8}(T), \quad t \in\left[t_{0}, T\right] \cap\left[t_{0}, T_{m}\right) .
$$

Hence,

$$
\left\|v\left(t_{2}\right)-v\left(t_{1}\right)\right\|_{W_{3}^{1}(\Omega)} \leq \int_{t_{1}}^{t_{2}}\left\|\partial_{t} v(s)\right\|_{W_{3}^{1}(\Omega)} \mathrm{d} s \leq C(T)\left(t_{2}-t_{1}\right) .
$$

Furthermore, in view of Hölder's inequality, we obtain from Lemma 3.3 and Corollary 3.7

$$
\begin{aligned}
\left\|w\left(t_{2}\right)-w\left(t_{1}\right)\right\|_{W_{3}^{1}(\Omega)} & \leq C\left\|w\left(t_{2}\right)-w\left(t_{1}\right)\right\|_{W_{\infty}^{1}(\Omega)}^{1 / 3}\left\|w\left(t_{2}\right)-w\left(t_{1}\right)\right\|_{W_{2}^{1}(\Omega)}^{2 / 3} \\
& \leq C\left(\left\|w\left(t_{1}\right)\right\|_{W_{\infty}^{1}(\Omega)}+\left\|w\left(t_{2}\right)\right\|_{W_{\infty}^{1}(\Omega)}\right)^{1 / 3}\left(\int_{t_{1}}^{t_{2}}\left\|\partial_{t} w(s)\right\|_{W_{2}^{1}(\Omega)} \mathrm{d} s\right)^{2 / 3} \\
& \leq C\left(2 C_{5}(T)\right)^{1 / 3}\left(\int_{t_{1}}^{t_{2}}\left\|\partial_{t} w(s)\right\|_{W_{2}^{1}(\Omega)}^{2} \mathrm{~d} s\right)^{1 / 3}\left(t_{2}-t_{1}\right)^{1 / 3} \\
& \leq C(T)\left(C_{1}(T)\right)^{1 / 3}\left(t_{2}-t_{1}\right)^{1 / 3} .
\end{aligned}
$$

Since $W_{2}^{4 / 3}(\Omega)$ is continuously embedded in $W_{3}^{1}(\Omega)$, interpolation inequalities (see [3, Theorem 7.2]), Hölder's inequality, Lemma 3.8, and Corollary 3.9 yield

$$
\begin{aligned}
\left\|u\left(t_{2}\right)-u\left(t_{1}\right)\right\|_{W_{3}^{1}(\Omega)} & \leq C\left\|u\left(t_{2}\right)-u\left(t_{1}\right)\right\|_{W_{2}^{4 / 3}(\Omega)} \\
& \leq C\left\|u\left(t_{2}\right)-u\left(t_{1}\right)\right\|_{W_{2}^{2 \gamma}(\Omega)}^{2 / 3 \gamma}\left\|u\left(t_{2}\right)-u\left(t_{1}\right)\right\|_{L_{2}(\Omega)}^{2 \delta} \\
& \leq C\left(\left\|u\left(t_{1}\right)\right\|_{W_{2}^{2 \gamma}(\Omega)}+\left\|u\left(t_{2}\right)\right\|_{W_{2}^{2 \gamma}(\Omega)}\right)^{2 / 3 \gamma}\left(\int_{t_{1}}^{t_{2}}\left\|\partial_{t} u(s)\right\|_{L_{2}(\Omega)} \mathrm{d} s\right)^{2 \delta} \\
& \leq C\left(2 C_{7}(T)\right)^{2 / 3 \gamma}\left(\int_{t_{1}}^{t_{2}}\left\|\partial_{t} u(s)\right\|_{L_{2}(\Omega)}^{2} \mathrm{~d} s\right)^{\delta}\left(t_{2}-t_{1}\right)^{\delta} \\
& \leq C(T)\left(C_{6}(T)\right)^{\delta}\left(t_{2}-t_{1}\right)^{\delta} .
\end{aligned}
$$


Combining (3.24), (3.25), and (3.26) completes the proof, since $0<\delta<1 / 3$.

Proof of Theorem 1.1. Proposition 3.1 and Corollary 3.2 imply the claim as soon as we know that $T_{m}=\infty$. Since $(u, v, w) \in B U C\left(\left[0, t_{0}\right] ; W_{3}^{1}\left(\Omega ; \mathbb{R}^{3}\right)\right)$ by Proposition 3.1, (3.23) and Lemma 3.10 ensure that (3.2) is satisfied for any $T>0$, and we indeed conclude that $T_{m}=\infty$.

\section{Bounded SOLUtions FOR SMALL VALUES OF $M$}

Let $M>0$. In this section, we assume that

$$
\left(u_{0}, v_{0}, w_{0}\right) \in \mathcal{I}_{M},
$$

and denote the corresponding global solution to (1.1)-(1.2) by $(u, v, w)$, see Theorem 1.1. Throughout this section, $b$ and $\left(b_{i}\right)_{i \geq 1}$ denote positive constants depending only on $\Omega, \theta, D, \alpha, u_{0}, v_{0}$, and $w_{0}$. Dependence upon additional parameters is indicated explicitly.

In order to show the boundedness of the solution to (1.1)-(1.2), we first have a look at the evolution of the $L_{1}$-norms of $(u, v, w)$.

Lemma 4.1. For all $t \geq 0$,

$$
\begin{aligned}
& \|(u+v)(t)\|_{L_{1}(\Omega)}=M=\left\|u_{0}+v_{0}\right\|_{L_{1}(\Omega)}, \\
& \|u(t)\|_{L_{1}(\Omega)} \leq M \quad \text { and } \quad\|v(t)\|_{L_{1}(\Omega)} \leq M, \\
& \|w(t)\|_{L_{1}(\Omega)} \leq\left\|w_{0}\right\|_{L_{1}(\Omega)}+\frac{M}{\alpha} .
\end{aligned}
$$

Proof. The identity (4.2) is nothing but (1.6), and (4.3) is a consequence thereof in view of the nonnegativity of $u$ and $v$. Using the nonnegativity of $v$ and $w$ as well as (1.1c), (1.1d), and (4.3), we obtain

$$
\frac{\mathrm{d}}{\mathrm{d} t}\|w\|_{L_{1}(\Omega)}+\alpha\|w\|_{L_{1}(\Omega)}=\|v\|_{L_{1}(\Omega)} \leq M
$$

Hence

$$
\|w(t)\|_{L_{1}(\Omega)} \leq e^{-\alpha t}\left\|w_{0}\right\|_{L_{1}(\Omega)}+M \int_{0}^{t} e^{-\alpha(t-s)} \mathrm{d} s \leq\left\|w_{0}\right\|_{L_{1}(\Omega)}+\frac{M}{\alpha}
$$

for all $t \geq 0$ as $\alpha>0$.

Next, as in $[6,10,21]$, we use the structure of the Liapunov functional $\mathcal{L}$ defined in $(2.2)$ and follow the strategy from [17] (see also [8]). We begin with a lower bound on $\mathcal{L}$ for $M$ appropriately small which relies on the Trudinger-Moser inequality and first note the following consequence thereof (see [7, Proposition 2.3] and [21, Section 2]).

Proposition 4.2. There is $K_{0}>0$ depending only on $\Omega$ such that

$$
\int_{\Omega} e^{|z|} \mathrm{d} x \leq K_{0} \exp \left(\frac{\|\nabla z\|_{L_{2}(\Omega)}^{2}}{8 \pi}+\frac{\|z\|_{L_{1}(\Omega)}}{|\Omega|}\right) \quad \text { for all } z \in W_{2}^{1}(\Omega) .
$$


Lemma 4.3. Assume that (4.1) is satisfied. There is $b_{1}>0$ such that, for all $t \geq 0$,

$$
\begin{aligned}
\mathcal{L}(u(t), v(t), w(t)) \leq & \mathcal{L}\left(u_{0}, v_{0}, w_{0}\right)<\infty \\
\mathcal{L}(u(t), v(t), w(t)) \geq & \frac{4 \pi(1+\theta) D-M}{8 \pi}\|\nabla w(t)\|_{L_{2}(\Omega)}^{2}+\frac{\alpha(1+\theta)}{2}\|w(t)\|_{L_{2}(\Omega)}^{2} \\
& +\frac{1}{2}\|(D \Delta w-\alpha w+v)(t)\|_{L_{2}(\Omega)}^{2}-b_{1} .
\end{aligned}
$$

Proof. We first observe that (4.1) guarantees that $\mathcal{L}\left(u_{0}, v_{0}, w_{0}\right) \in \mathbb{R}$, while Theorem 1.1 ensures that $\mathcal{L}(u(t), v(t), w(t)) \in \mathbb{R}$ for all $t>0$.

Next, using the convexity of $s \mapsto-\ln (s)$ and Jensen's inequality as well as the nonnegativity of $u$ and $v$, we obtain

$$
\begin{aligned}
0 & =-\|u\|_{L_{1}(\Omega)} \ln \left(\int_{\Omega} \frac{u}{\|u\|_{L_{1}(\Omega)}} \frac{e^{w}\|u\|_{L_{1}(\Omega)}}{u\left\|e^{w}\right\|_{L_{1}(\Omega)}} \mathrm{d} x\right) \\
& \leq-\|u\|_{L_{1}(\Omega)} \int_{\Omega} \frac{u}{\|u\|_{L_{1}(\Omega)}} \ln \left(\frac{e^{w}\|u\|_{L_{1}(\Omega)}}{u\left\|e^{w}\right\|_{L_{1}(\Omega)}}\right) \mathrm{d} x \\
& =\int_{\Omega}(u \ln u-u w) \mathrm{d} x+\|u\|_{L_{1}(\Omega)} \ln \left(\left\|e^{w}\right\|_{L_{1}(\Omega)}\right)-\|u\|_{L_{1}(\Omega)} \ln \left(\|u\|_{L_{1}(\Omega)}\right),
\end{aligned}
$$

as well as

$$
\begin{aligned}
0 & =-\|v\|_{L_{1}(\Omega)} \ln \left(\int_{\Omega} \frac{v}{\|v\|_{L_{1}(\Omega)}} \frac{e^{w}\|\theta v\|_{L_{1}(\Omega)}}{\theta v\left\|e^{w}\right\|_{L_{1}(\Omega)}} \mathrm{d} x\right) \\
& \leq-\|v\|_{L_{1}(\Omega)} \int_{\Omega} \frac{v}{\|v\|_{L_{1}(\Omega)}} \ln \left(\frac{e^{w}\|\theta v\|_{L_{1}(\Omega)}}{\theta v\left\|e^{w}\right\|_{L_{1}(\Omega)}}\right) \mathrm{d} x \\
& =\int_{\Omega}(v \ln (\theta v)-v w) \mathrm{d} x+\|v\|_{L_{1}(\Omega)} \ln \left(\left\|e^{w}\right\|_{L_{1}(\Omega)}\right)-\|v\|_{L_{1}(\Omega)} \ln \left(\|\theta v\|_{L_{1}(\Omega)}\right) .
\end{aligned}
$$

In view of the nonnegativity of $(u, v, w), L$, and $L_{\theta}$, we infer from (2.2), (4.2), (4.7), (4.8), and Proposition 4.2 that

$$
\begin{aligned}
\mathcal{L}(u, v, w)= & \int_{\Omega}(u \ln u-u w) \mathrm{d} x+\int_{\Omega}(v \ln (\theta v)-v w) \mathrm{d} x-\|u\|_{L_{1}(\Omega)}+|\Omega|-\|v\|_{L_{1}(\Omega)}+\frac{|\Omega|}{\theta} \\
& +\frac{1+\theta}{2}\left(D\|\nabla w\|_{L_{2}(\Omega)}^{2}+\alpha\|w\|_{L_{2}(\Omega)}^{2}\right)+\frac{1}{2}\|D \Delta w-\alpha w+v\|_{L_{2}(\Omega)}^{2} \\
\geq & -M \ln \left(\left\|e^{w}\right\|_{L_{1}(\Omega)}\right)+L\left(\|u\|_{L_{1}(\Omega)}\right)+L_{\theta}\left(\|v\|_{L_{1}(\Omega)}\right)+(|\Omega|-1)\left(1+\frac{1}{\theta}\right) \\
& +\frac{1+\theta}{2}\left(D\|\nabla w\|_{L_{2}(\Omega)}^{2}+\alpha\|w\|_{L_{2}(\Omega)}^{2}\right)+\frac{1}{2}\|D \Delta w-\alpha w+v\|_{L_{2}(\Omega)}^{2} \\
\geq & \frac{4 \pi(1+\theta) D-M}{8 \pi}\|\nabla w\|_{L_{2}(\Omega)}^{2}+\frac{\alpha(1+\theta)}{2}\|w\|_{L_{2}(\Omega)}^{2}+\frac{1}{2}\|D \Delta w-\alpha w+v\|_{L_{2}(\Omega)}^{2}
\end{aligned}
$$




$$
-M \ln K_{0}-\frac{M}{|\Omega|}\|w\|_{L_{1}(\Omega)}+(|\Omega|-1)\left(1+\frac{1}{\theta}\right) .
$$

Inserting (4.4), we obtain (4.6), while (4.5) immediately follows from (2.3) since $\mathcal{D} \geq 0$.

Hence, for $M \in(0,4 \pi(1+\theta) D)$, the Liapunov functional is bounded from below and we obtain further refined estimates.

Lemma 4.4. Assume that (4.1) is satisfied with $M \in(0,4 \pi(1+\theta) D)$. There is $b_{2}>0$ such that, for all $t \geq 0$,

$$
\begin{array}{r}
\|u(t) \ln (u(t))\|_{L_{1}(\Omega)}+\|v(t) \ln (v(t))\|_{L_{1}(\Omega)}+\|w(t)\|_{W_{2}^{1}(\Omega)}+\left\|\partial_{t} w(t)\right\|_{L_{2}(\Omega)} \leq b_{2}, \\
\int_{0}^{\infty}\left\|\partial_{t} w(s)\right\|_{W_{2}^{1}(\Omega)}^{2} \mathrm{~d} s \leq b_{2} .
\end{array}
$$

Proof. Since (1.1c) and Lemma 4.3 imply that

$$
\min \left\{\frac{4 \pi(1+\theta) D-M}{8 \pi}, \frac{\alpha(1+\theta)}{2}, \frac{1}{2}\right\}\left(\|w(t)\|_{W_{2}^{1}(\Omega)}^{2}+\left\|\partial_{t} w(t)\right\|_{L_{2}(\Omega)}^{2}\right) \leq b_{1}+\mathcal{L}\left(u_{0}, v_{0}, w_{0}\right)
$$

and $M \in(0,4 \pi(1+\theta) D)$, we get

$$
\|w(t)\|_{W_{2}^{1}(\Omega)}+\left\|\partial_{t} w(t)\right\|_{L_{2}(\Omega)} \leq b, \quad t \geq 0 .
$$

Next, (2.2), (4.2), (4.3), (4.5), and the Young inequality $A B \leq e^{A-1}+B \ln B$ for $A, B>0$ yield

$$
\begin{aligned}
& \int_{\Omega}(u \ln u+v \ln (\theta v)) \mathrm{d} x \\
& \quad \leq \mathcal{L}(u, v, w)+\int_{\Omega}(u+v) \mathrm{d} x+\int_{\Omega}(u+v) w \mathrm{~d} x \\
& \quad \leq \mathcal{L}\left(u_{0}, v_{0}, w_{0}\right)+M+\int_{\Omega}\left(\frac{u}{2} \ln \left(\frac{u}{2}\right)+\frac{v}{2} \ln \left(\frac{v}{2}\right)+2 e^{2 w-1}\right) \mathrm{d} x \\
& \quad \leq \mathcal{L}\left(u_{0}, v_{0}, w_{0}\right)+M+\frac{1}{2} \int_{\Omega}(u \ln u+v \ln (\theta v)) \mathrm{d} x+2 \int_{\Omega} e^{2 w-1} \mathrm{~d} x-\frac{\ln \theta}{2} \int_{\Omega} v \mathrm{~d} x \\
& \quad \leq \mathcal{L}\left(u_{0}, v_{0}, w_{0}\right)+\frac{1}{2} \int_{\Omega}(u \ln u+v \ln (\theta v)) \mathrm{d} x+2 \int_{\Omega} e^{2 w} \mathrm{~d} x+M(1+|\ln \theta|) .
\end{aligned}
$$

In view of Proposition 4.2 this implies

$$
\int_{\Omega}(u \ln u+v \ln (\theta v)) \mathrm{d} x \leq 4 K_{0} \exp \left(\frac{\|\nabla w\|_{L_{2}(\Omega)}^{2}}{2 \pi}+2 \frac{\|w\|_{L_{1}(\Omega)}}{|\Omega|}\right)+b,
$$

so that, in view of $z \ln z \geq-1 / e$ for all $z \geq 0$, we conclude from (4.3), (4.4), and (4.10) that

$$
\begin{aligned}
\|u \ln u\|_{L_{1}(\Omega)} & +\|v \ln v\|_{L_{1}(\Omega)} \\
& \leq \int_{\Omega}(u \ln u+v \ln v) \mathrm{d} x+\frac{4|\Omega|}{e}
\end{aligned}
$$




$$
\begin{aligned}
& \leq \int_{\Omega}(u \ln u+v \ln (\theta v)) \mathrm{d} x-\ln \theta \int_{\Omega} v \mathrm{~d} x+\frac{4|\Omega|}{e} \\
& \leq \int_{\Omega}(u \ln u+v \ln (\theta v)) \mathrm{d} x+M|\ln \theta|+\frac{4|\Omega|}{e} \leq b, \quad t \geq 0 .
\end{aligned}
$$

Finally, we deduce from (1.1c), (2.5), and Lemmas 2.1 and 4.3 that

$$
\begin{aligned}
\min \{D, 1+\theta+\alpha\} \int_{0}^{t}\left\|\partial_{t} w(s)\right\|_{W_{2}^{1}(\Omega)}^{2} \mathrm{~d} s & \leq \int_{0}^{t} \mathcal{D}(u(s), v(s), w(s)) \mathrm{d} s \\
& \leq \mathcal{L}\left(u_{0}, v_{0}, w_{0}\right)-\mathcal{L}(u(t), v(t), w(t)) \\
& \leq \mathcal{L}\left(u_{0}, v_{0}, w_{0}\right)+b_{1}
\end{aligned}
$$

which, in view of (4.10) and (4.11), completes the proof.

Starting from the previous estimates, we derive further time-independent estimates for the solution by using the ideas from [17] as well as the following inequality, which is [5, Equation (22)]:

Given $\eta>0$, there is a positive constant $\kappa_{\eta}$ depending only on $\eta$ and $\Omega$ such that

$$
\|z\|_{L_{3}(\Omega)}^{3} \leq \eta\|z\|_{W_{2}^{1}(\Omega)}^{2}\|z \ln |z|\|_{L_{1}(\Omega)}+\kappa_{\eta}\|z\|_{L_{1}(\Omega)} \quad \text { for all } z \in W_{2}^{1}(\Omega) .
$$

Lemma 4.5. Assume that (4.1) is satisfied with $M \in(0,4 \pi(1+\theta) D)$. There is $b_{3}>0$ such that, for all $t \geq 0$,

$$
\|u(t)\|_{L_{2}(\Omega)}+\|v(t)\|_{L_{3}(\Omega)} \leq b_{3} .
$$

Proof. It follows from (1.1), (4.3), Hölder's and Young's inequalities, and the nonnegativity of $u$ and $w$

$$
\begin{aligned}
\frac{\mathrm{d}}{\mathrm{d} t}\|u\|_{L_{2}(\Omega)}^{2} & +2\|\nabla u\|_{L_{2}(\Omega)}^{2}=\int_{\Omega}\left(-u^{2} \Delta w+2 \theta u v-2 u^{2}\right) \mathrm{d} x \\
& \leq \frac{1}{D} \int_{\Omega} u^{2}\left(v-\alpha w-\partial_{t} w\right) \mathrm{d} x-2\left\|u-\frac{\theta v}{2}\right\|_{L_{2}(\Omega)}^{2}+\frac{\theta^{2}}{2}\|v\|_{L_{2}(\Omega)}^{2} \\
& \leq \frac{1}{D}\|v\|_{L_{3}(\Omega)}\|u\|_{L_{3}(\Omega)}^{2}+\frac{1}{D}\left\|\partial_{t} w\right\|_{L_{2}(\Omega)}\|u\|_{L_{4}(\Omega)}^{2}+\frac{2 \theta}{3}\|v\|_{L_{3}(\Omega)}^{3}+\frac{\theta^{4}}{3} \\
& \leq \theta\|v\|_{L_{3}(\Omega)}^{3}+\frac{2}{3 \theta^{1 / 2} D^{3 / 2}}\|u\|_{L_{3}(\Omega)}^{3}+\frac{1}{D}\left\|\partial_{t} w\right\|_{L_{2}(\Omega)}\|u\|_{L_{4}(\Omega)}^{2}+b .
\end{aligned}
$$

Next, Gagliardo-Nirenberg's inequality yields

$$
\begin{aligned}
\frac{\mathrm{d}}{\mathrm{d} t}\|u\|_{L_{2}(\Omega)}^{2}+2\|\nabla u\|_{L_{2}(\Omega)}^{2} \leq & \theta\|v\|_{L_{3}(\Omega)}^{3}+b\left(1+\|u\|_{L_{3}(\Omega)}^{3}\right) \\
& +b\left\|\partial_{t} w\right\|_{L_{2}(\Omega)}\left(\|\nabla u\|_{L_{2}(\Omega)}\|u\|_{L_{2}(\Omega)}+\|u\|_{L_{2}(\Omega)}^{2}\right) \\
\leq & \theta\|v\|_{L_{3}(\Omega)}^{3}+b\left(1+\|u\|_{L_{3}(\Omega)}^{3}\right)+\frac{1}{2}\|\nabla u\|_{L_{2}(\Omega)}^{2} \\
& +b\left(\left\|\partial_{t} w\right\|_{L_{2}(\Omega)}+\left\|\partial_{t} w\right\|_{L_{2}(\Omega)}^{2}\right)\|u\|_{L_{2}(\Omega)}^{2} .
\end{aligned}
$$


In view of Lemma 4.4, this implies

$$
\frac{\mathrm{d}}{\mathrm{d} t}\|u\|_{L_{2}(\Omega)}^{2}+\frac{3}{2}\|\nabla u\|_{L_{2}(\Omega)}^{2} \leq \theta\|v\|_{L_{3}(\Omega)}^{3}+b\left(1+\|u\|_{L_{3}(\Omega)}^{3}+b_{2}\left(1+b_{2}\right)\|u\|_{L_{2}(\Omega)}^{2}\right) .
$$

Moreover, using (1.1b) along with Young's inequality, we obtain

$$
\frac{1}{3} \frac{\mathrm{d}}{\mathrm{d} t}\|v\|_{L_{3}(\Omega)}^{3}+\theta\|v\|_{L_{3}(\Omega)}^{3}=\int_{\Omega} u v^{2} \mathrm{~d} x \leq \frac{4}{3 \theta^{2}}\|u\|_{L_{3}(\Omega)}^{3}+\frac{\theta}{3}\|v\|_{L_{3}(\Omega)}^{3} .
$$

Hence,

$$
\frac{\mathrm{d}}{\mathrm{d} t}\|v\|_{L_{3}(\Omega)}^{3}+2 \theta\|v\|_{L_{3}(\Omega)}^{3} \leq \frac{4}{\theta^{2}}\|u\|_{L_{3}(\Omega)}^{3} .
$$

Defining $Y:=\|u\|_{L_{2}(\Omega)}^{2}+\|v\|_{L_{3}(\Omega)}^{3}$, we deduce from (4.13), (4.14) along with (4.3), (4.12), and Lemma 4.4 that, for $\eta>0$,

$$
\begin{aligned}
\frac{\mathrm{d} Y}{\mathrm{~d} t}+\frac{3}{2}\|\nabla u\|_{L_{2}(\Omega)}^{2}+\theta\|v\|_{L_{3}(\Omega)}^{3} & \leq b\left(1+\|u\|_{L_{3}(\Omega)}^{3}+\|u\|_{L_{2}(\Omega)}^{2}\right) \\
& \leq b\left(1+\eta b_{2}\|u\|_{W_{2}^{1}(\Omega)}^{2}+\kappa_{\eta} M+\|u\|_{L_{2}(\Omega)}^{2}\right) \\
& \leq \eta b_{4}\|\nabla u\|_{L_{2}(\Omega)}^{2}+b\left(1+\|u\|_{L_{2}(\Omega)}^{2}+\kappa_{\eta}\right) .
\end{aligned}
$$

Choosing $\eta:=1 / 2 b_{4}>0$, we find

$$
\frac{\mathrm{d} Y}{\mathrm{~d} t}+\|\nabla u\|_{L_{2}(\Omega)}^{2}+\theta\|v\|_{L_{3}(\Omega)}^{3} \leq b\left(1+\|u\|_{L_{2}(\Omega)}^{2}\right) .
$$

Owing to the Gagliardo-Nirenberg and Young inequalities along with (4.3),

$$
\begin{aligned}
\|u\|_{L_{2}(\Omega)}^{4} & \leq b\left(\|\nabla u\|_{L_{2}(\Omega)}^{2} M^{2}+M^{4}\right), \\
\|u\|_{L_{2}(\Omega)}^{2} & \leq b_{5}\|u\|_{L_{2}(\Omega)}^{4}+\frac{1}{b_{5}},
\end{aligned}
$$

so that, using once more Young's inequality,

$$
\begin{aligned}
\frac{\mathrm{d} Y}{\mathrm{~d} t}+\|u\|_{L_{2}(\Omega)}^{2}+b_{5}\|u\|_{L_{2}(\Omega)}^{4}+\theta\|v\|_{L_{3}(\Omega)}^{3} & \leq \frac{\mathrm{d} Y}{\mathrm{~d} t}+2 b_{5}\|u\|_{L_{2}(\Omega)}^{4}+\theta\|v\|_{L_{3}(\Omega)}^{3}+\frac{1}{b_{5}} \\
& \leq b\left(1+\|u\|_{L_{2}(\Omega)}^{2}\right)+\frac{1}{b_{5}} \leq b_{5}\|u\|_{L_{2}(\Omega)}^{4}+b .
\end{aligned}
$$

Consequently,

$$
\frac{\mathrm{d} Y}{\mathrm{~d} t}+\min \{1, \theta\} Y \leq b, \quad t>0,
$$

from which Lemma 4.5 follows after integration with respect to time.

Corollary 4.6. Assume that (4.1) is satisfied with $M \in(0,4 \pi(1+\theta) D)$. There is $b_{6}>0$ such that, for all $t \geq 0$,

$$
\|\Delta w(t)\|_{L_{2}(\Omega)}+\|w(t)\|_{W_{3}^{1}(\Omega)} \leq b_{6}
$$


Proof. First, by (1.1c), Lemma 4.4, and Lemma 4.5,

$$
\begin{aligned}
D\|\Delta w\|_{L_{2}(\Omega)} & =\left\|\partial_{t} w+\alpha w-v\right\|_{L_{2}(\Omega)} \leq\left\|\partial_{t} w\right\|_{L_{2}(\Omega)}+\|\alpha w\|_{L_{2}(\Omega)}+\|v\|_{L_{2}(\Omega)} \\
& \leq(1+\alpha) b_{2}+|\Omega|^{1 / 6} b_{3} .
\end{aligned}
$$

Next, let $t>0$. We infer from (1.1c), Duhamel's formula, the regularizing effect of the heat semigroup, and Lemma 4.5 that

$$
\begin{aligned}
\|w(t)\|_{W_{3}^{1}(\Omega)} & \leq b e^{-\alpha t / 2}\left\|w_{0}\right\|_{W_{3}^{1}(\Omega)}+b \int_{0}^{t} e^{-\alpha(t-s) / 2}(t-s)^{-1 / 2}\|v(s)\|_{L_{3}(\Omega)} \mathrm{d} s \\
& \leq b\left(1+b_{3} \int_{0}^{\infty} e^{-\alpha s / 2} s^{-1 / 2} \mathrm{~d} s\right),
\end{aligned}
$$

which completes the proof.

The previous result allows us to obtain better $L_{p}$-estimates for $u$.

Lemma 4.7. Assume that (4.1) is satisfied with $M \in(0,4 \pi(1+\theta) D)$. For any $p \in[1, \infty)$, there is $b_{7}(p)>0$ such that, for all $t \geq 0$,

$$
\|u(t)\|_{L_{p}(\Omega)} \leq b_{7}(p) .
$$

Proof. We fix $p>2$. Using (1.1a), (1.1d), Hölder's inequality, and Corollary 4.6, we have

$$
\begin{aligned}
\frac{1}{p} \frac{\mathrm{d}}{\mathrm{d} t}\|u\|_{L_{p}(\Omega)}^{p} & =-(p-1) \int_{\Omega} u^{p-2}|\nabla u|^{2} \mathrm{~d} x+(p-1) \int_{\Omega} u^{p-1} \nabla u \cdot \nabla w \mathrm{~d} x+\int_{\Omega} u^{p-1}(\theta v-u) \mathrm{d} x \\
& =-\frac{4(p-1)}{p^{2}}\left\|\nabla\left(u^{p / 2}\right)\right\|_{L_{2}(\Omega)}^{2}-\frac{p-1}{p} \int_{\Omega} u^{p} \Delta w \mathrm{~d} x+\int_{\Omega} u^{p-1}(\theta v-u) \mathrm{d} x \\
& \leq-\frac{4(p-1)}{p^{2}}\left\|\nabla\left(u^{p / 2}\right)\right\|_{L_{2}(\Omega)}^{2}+\frac{p-1}{p} b_{6}\left\|u^{p / 2}\right\|_{L^{4}(\Omega)}^{2}+\int_{\Omega} u^{p-1}(\theta v-u) \mathrm{d} x .
\end{aligned}
$$

Also, it follows from (1.1b) and Young's inequality that

$$
\begin{aligned}
\frac{1+\theta^{p-1}}{p} \frac{\mathrm{d}}{\mathrm{d} t}\|v\|_{L_{p}(\Omega)}^{p} & =-\int_{\Omega}(\theta v)^{p-1}(\theta v-u) \mathrm{d} x-\theta\|v\|_{L_{p}(\Omega)}^{p}+\int_{\Omega} u v^{p-1} \mathrm{~d} x \\
& \leq-\int_{\Omega}(\theta v)^{p-1}(\theta v-u) \mathrm{d} x-\frac{\theta}{p}\|v\|_{L_{p}(\Omega)}^{p}+\frac{1}{p \theta^{p-1}}\|u\|_{L_{p}(\Omega)}^{p} .
\end{aligned}
$$

Next, in view of the Gagliardo-Nirenberg inequality (see, e.g., [19, Lemma 2.3] for a version involving $L_{q}$-spaces for $\left.q>0\right), p>2$, Lemma 4.5, and Young's inequality, we further obtain

$$
\begin{aligned}
\frac{p-1}{p} b_{6}\left\|u^{p / 2}\right\|_{L_{4}(\Omega)}^{2} & \leq b(p)\left(\left\|\nabla\left(u^{p / 2}\right)\right\|_{L_{2}(\Omega)}^{(p-1) / p}\left\|u^{p / 2}\right\|_{L_{4 / p}(\Omega)}^{1 / p}+\left\|u^{p / 2}\right\|_{L_{4 / p}(\Omega)}\right)^{2} \\
& \leq b(p)\left(\left\|\nabla\left(u^{p / 2}\right)\right\|_{L_{2}(\Omega)}^{2(p-1) / p}\|u\|_{L_{2}(\Omega)}+\|u\|_{L_{2}(\Omega)}^{p}\right) \\
& \leq b(p)\left(b_{3}\left\|\nabla\left(u^{p / 2}\right)\right\|_{L_{2}(\Omega)}^{2(p-1) / p}+b_{3}^{p}\right)
\end{aligned}
$$




$$
\leq \frac{2(p-1)}{p^{2}}\left\|\nabla\left(u^{p / 2}\right)\right\|_{L_{2}(\Omega)}^{2}+b(p) .
$$

Combining (4.15), (4.16), and (4.17) and using the monotonicity of $z \mapsto z^{p-1}$ on $[0, \infty)$, we deduce that

$$
\frac{1}{p} \frac{\mathrm{d} y}{\mathrm{~d} t}+\frac{2(p-1)}{p^{2}}\left\|\nabla\left(u^{p / 2}\right)\right\|_{L_{2}(\Omega)}^{2}+\frac{\theta}{p}\|v\|_{L_{p}(\Omega)}^{p} \leq b_{8}(p)\left(1+\|u\|_{L_{p}(\Omega)}^{p}\right),
$$

with $y:=\|u\|_{L_{p}(\Omega)}^{p}+\left(1+\theta^{p-1}\right)\|v\|_{L_{p}(\Omega)}^{p}$. Using once more Lemma 4.5 and Gagliardo-Nirenberg's and Young's inequalities gives

$$
\begin{aligned}
\|u\|_{L^{p}(\Omega)}^{p} & =\left\|u^{p / 2}\right\|_{L_{2}(\Omega)}^{2} \leq b(p)\left(\left\|\nabla\left(u^{p / 2}\right)\right\|_{L_{2}(\Omega)}^{(p-2) / p}\left\|u^{p / 2}\right\|_{L_{4 / p}(\Omega)}^{2 / p}+\left\|u^{p / 2}\right\|_{L_{4 / p}(\Omega)}\right)^{2} \\
& \leq b(p)\left(\left\|\nabla\left(u^{p / 2}\right)\right\|_{L_{2}(\Omega)}^{2(p-2) / p}\left\|u^{p / 2}\right\|_{L_{4 / p}(\Omega)}^{4 / p}+\left\|u^{p / 2}\right\|_{L_{4 / p}(\Omega)}^{2}\right) \\
& \leq b(p)\left(b_{3}^{2}\left\|\nabla\left(u^{p / 2}\right)\right\|_{L_{2}(\Omega)}^{2(p-2) / p}+b_{3}^{p}\right) \\
& \leq \frac{(p-1)}{p^{2} b_{8}(p)}\left\|\nabla\left(u^{p / 2}\right)\right\|_{L_{2}(\Omega)}^{2}+b(p),
\end{aligned}
$$

and we infer from (4.18) that

$$
\begin{aligned}
& \frac{1}{p} \frac{\mathrm{d} y}{\mathrm{~d} t}+\frac{p-1}{p^{2}}\left\|\nabla\left(u^{p / 2}\right)\right\|_{L_{2}(\Omega)}^{2}+b_{8}(p)\|u\|_{L^{p}(\Omega)}^{p}+\frac{\theta}{p}\|v\|_{L_{p}(\Omega)}^{p} \\
& \leq \frac{1}{p} \frac{\mathrm{d} y}{\mathrm{~d} t}+\frac{2(p-1)}{p^{2}}\left\|\nabla\left(u^{p / 2}\right)\right\|_{L_{2}(\Omega)}^{2}+\frac{\theta}{p}\|v\|_{L_{p}(\Omega)}^{p}+b(p) \\
& \leq b_{8}(p)\left(1+\|u\|_{L_{p}(\Omega)}^{p}\right)+b(p) \leq \frac{(p-1)}{p^{2}}\left\|\nabla\left(u^{p / 2}\right)\right\|_{L_{2}(\Omega)}^{2}+b(p) .
\end{aligned}
$$

Hence,

$$
\frac{\mathrm{d} y}{\mathrm{~d} t}+\min \left\{p b_{8}(p), \frac{\theta}{1+\theta^{p-1}}\right\} y \leq b(p), \quad t \geq 0 .
$$

Integration with respect to time of the above differential inequality completes the proof, after noticing that $u_{0} \in L_{p}(\Omega)$ due to the continuous embedding of $W_{3}^{1}(\Omega)$ in $L_{\infty}(\Omega)$.

We are now in a position to prove Theorem 1.2.

Proof of Theorem 1.2. (a) We fix $p \in(2,3)$ and $\xi \in(2 / p, 1)$. Since

$$
\left(W_{p, \mathcal{B}}^{-1}(\Omega), W_{p, \mathcal{B}}^{1}(\Omega)\right)_{(1+\xi) / 2, p} \doteq W_{p, \mathcal{B}}^{\xi}(\Omega)
$$

by $[3$, Theorem 7.2$]$ and writing (1.1a) as

$$
\partial_{t} u-\Delta u+u=\theta v-\operatorname{div}(u \nabla w) \quad \text { in }(0, \infty) \times \Omega,
$$


we infer from Duhamel's formula and [4, Theorem V.2.1.3] that, for $t \geq 0$,

$$
\begin{aligned}
\|u(t)\|_{W_{p}^{\xi}(\Omega)} & \leq b e^{-t / 2}\left\|u_{0}\right\|_{W_{p}^{\xi}(\Omega)}+b \int_{0}^{t}(t-s)^{-(1+\xi) / 2} e^{-(t-s) / 2}\|\theta v(s)-\operatorname{div}(u \nabla w)(s)\|_{W_{p, \mathcal{B}}^{-1}(\Omega)} \mathrm{d} s \\
& \leq b\left\|u_{0}\right\|_{W_{3}^{1}(\Omega)}+b \int_{0}^{t}(t-s)^{-(1+\xi) / 2} e^{-(t-s) / 2}\left(\|v(s)\|_{L_{p}(\Omega)}+\|(u \nabla w)(s)\|_{L_{p}(\Omega)}\right) \mathrm{d} s .
\end{aligned}
$$

We next deduce from Lemma 4.5, Corollary 4.6, Lemma 4.7, and Hölder's inequality that

$$
\begin{aligned}
\|u(t)\|_{W_{p}^{\xi}(\Omega)} \leq b+b \int_{0}^{t}(t-s)^{-(1+\xi) / 2} e^{-(t-s) / 2}\left(|\Omega|^{(3-p) / 3}\|v(s)\|_{L_{3}(\Omega)}\right. & \\
& \left.+\|u(s)\|_{L_{3 p /(3-p)}(\Omega)}\|\nabla w(s)\|_{L_{3}(\Omega)}\right) \mathrm{d} s \\
& \leq b+b\left[b_{3}+b_{6} b_{7}(3 p /(3-p))\right] \int_{0}^{t} s^{-(1+\xi) / 2} e^{-s / 2} \mathrm{~d} s \leq b .
\end{aligned}
$$

Since $\xi>2 / p$, the space $W_{p}^{\xi}(\Omega)$ is continuously embedded in $L_{\infty}(\Omega)$ and we deduce from the above estimate that

$$
\|u(t)\|_{L_{\infty}(\Omega)} \leq b_{9}, \quad t \geq 0 .
$$

Moreover, from (1.1b) and the comparison principle, we obtain

$$
\|v(t)\|_{L_{\infty}(\Omega)} \leq \max \left\{\frac{b_{9}}{\theta},\left\|v_{0}\right\|_{L_{\infty}(\Omega)}\right\}, \quad t \geq 0,
$$

which, together with Corollary 4.6 and the continuous embedding of $W_{3}^{1}(\Omega)$ in $L_{\infty}(\Omega)$, completes the proof of part (a).

(b) As $w$ is radially symmetric, an improved version of Proposition 4.2 is valid. Namely, in view of $[21$, Theorem 2.1], for any $\eta>0$, there is $K(\eta)>0$ depending only on $\eta$ and $\Omega$ such that

$$
\int_{\Omega} e^{w} \mathrm{~d} x \leq K(\eta) \exp \left(\left(\eta+\frac{1}{16 \pi}\right)\|\nabla w\|_{L_{2}(\Omega)}^{2}+\frac{2\|w\|_{L_{1}(\Omega)}}{|\Omega|}\right) .
$$

We then proceed as in the derivation of (4.9) with the help of this estimate with $\eta=(8 \pi(1+\theta) D-$ $M) /(32 \pi M)$ and (4.4) to deduce that

$$
\begin{aligned}
\mathcal{L}(u, v, w) \geq & -M \ln \left(\left\|e^{w}\right\|_{L_{1}(\Omega)}\right)+L\left(\|u\|_{L_{1}(\Omega)}\right)+L_{\theta}\left(\|v\|_{L_{1}(\Omega)}\right)+(|\Omega|-1)\left(1+\frac{1}{\theta}\right) \\
& +\frac{1+\theta}{2}\left(D\|\nabla w\|_{L_{2}(\Omega)}^{2}+\alpha\|w\|_{L_{2}(\Omega)}^{2}\right)+\frac{1}{2}\|D \Delta w-\alpha w+v\|_{L_{2}(\Omega)}^{2} \\
\geq & \frac{8 \pi(1+\theta) D-(1+16 \pi \eta) M}{16 \pi}\|\nabla w\|_{L_{2}(\Omega)}^{2}+\frac{\alpha(1+\theta)}{2}\|w\|_{L_{2}(\Omega)}^{2} \\
& +\frac{1}{2}\|D \Delta w-\alpha w+v\|_{L_{2}(\Omega)}^{2}-M \ln (K(\eta))-\frac{2 M}{|\Omega|}\|w\|_{L_{1}(\Omega)}+(|\Omega|-1)\left(1+\frac{1}{\theta}\right)
\end{aligned}
$$




$$
\geq \frac{8 \pi(1+\theta) D-M}{32 \pi}\|\nabla w\|_{L_{2}(\Omega)}^{2}+\frac{\alpha(1+\theta)}{2}\|w\|_{L_{2}(\Omega)}^{2}+\frac{1}{2}\|D \Delta w-\alpha w+v\|_{L_{2}(\Omega)}^{2}-b .
$$

Using this improved version of (4.6) in the remaining part of Section 4, we finish the proof of part (b).

\section{UNBOUNDED SOLUTIONS FOR LARGE MASS}

Given $M>0$, we denote by $\mathcal{S}_{M}$ the set of nonnegative stationary solutions $\left(u_{*}, v_{*}, w_{*}\right) \in W_{2, \mathcal{B}}^{2}\left(\Omega ; \mathbb{R}^{3}\right)$ to (1.1) satisfying $\left\|u_{*}+v_{*}\right\|_{L_{1}(\Omega)}=M$. In view of $(1.1 \mathrm{~b})$, this requires $u_{*}=\theta v_{*}$, which implies, together with (1.1a), that

$$
u_{*}=c \frac{e^{w_{*}}}{\left\|e^{w_{*}}\right\|_{L_{1}(\Omega)}}
$$

for some $c>0$, which is determined by the mass constraint. Hence, we define $\mathcal{S}_{M}$ in the following way:

$$
\begin{aligned}
\left(u_{*}, v_{*}, w_{*}\right) \in & \mathcal{S}_{M} \text { if } \\
& \left(u_{*}, v_{*}, w_{*}\right) \in W_{2, \mathcal{B}}^{2}\left(\Omega ; \mathbb{R}^{3}\right), \quad u_{*}, v_{*}, w_{*} \geq 0 \text { in } \Omega, \\
& u_{*}=\frac{\theta M}{\theta+1} \frac{e^{w_{*}}}{\left\|e^{w_{*}}\right\|_{L_{1}(\Omega)}}, \quad v_{*}=\frac{M}{\theta+1} \frac{e^{w_{*}}}{\left\|e^{w_{*}}\right\|_{L_{1}(\Omega)}}, \\
& -D \Delta w_{*}+\alpha w_{*}=\frac{M}{\theta+1} \frac{e^{w_{*}}}{\left\|e^{w_{*}}\right\|_{L_{1}(\Omega)}} \quad \text { in } \Omega, \quad \nabla w_{*} \cdot \mathbf{n}=0 \quad \text { on } \partial \Omega .
\end{aligned}
$$

As in $[12,13,29]$, we begin with a lower bound for the Liapunov function $\mathcal{L}$ on $\mathcal{S}_{M}$ for appropriate values of the mass $M$.

Proposition 5.1. (a) If $M \in(4 \pi(1+\theta) D, \infty) \backslash(4 \pi(1+\theta) D \mathbb{N})$, then

$$
\mu_{M}:=\inf _{\left(u_{*}, v_{*}, w_{*}\right) \in \mathcal{S}_{M}} \mathcal{L}\left(u_{*}, v_{*}, w_{*}\right)>-\infty .
$$

(b) If $\Omega=B_{R}(0)$ for some $R>0$ and $M \in(8 \pi(1+\theta) D, \infty)$, then

$$
\mu_{M}:=\inf _{\left(u_{*}, v_{*}, w_{*}\right) \in \mathcal{S}_{M, \text { rad }}} \mathcal{L}\left(u_{*}, v_{*}, w_{*}\right)>-\infty,
$$

where $\mathcal{S}_{M, \text { rad }}:=\left\{\left(u_{*}, v_{*}, w_{*}\right) \in \mathcal{S}_{M}: u_{*}, v_{*}, w_{*}\right.$ are radially symmetric $\}$.

Proof. (a) Let $\left(u_{*}, v_{*}, w_{*}\right) \in \mathcal{S}_{M}$. Then, in view of (5.2), (5.3), and the mass constraint $\| u_{*}+$ $v_{*} \|_{L_{1}(\Omega)}=M$, we deduce from (2.2) that

$$
\begin{aligned}
\mathcal{L}\left(u_{*}, v_{*}, w_{*}\right)= & \int_{\Omega}\left(u_{*} \ln u_{*}-u_{*}+1+v_{*} \ln \left(\theta v_{*}\right)-v_{*}+\frac{1}{\theta}-\left(u_{*}+v_{*}\right) w_{*}\right) \mathrm{d} x \\
& +\frac{1+\theta}{2}\left(D\left\|\nabla w_{*}\right\|_{L_{2}(\Omega)}^{2}+\alpha\left\|w_{*}\right\|_{L_{2}(\Omega)}^{2}\right)+\frac{1}{2}\left\|D \Delta w_{*}-\alpha w_{*}+v_{*}\right\|_{L_{2}(\Omega)}^{2} \\
= & \int_{\Omega}\left(\left(u_{*}+v_{*}\right) \ln u_{*}-\left(u_{*}+v_{*}\right) w_{*}\right) \mathrm{d} x-M+|\Omega|\left(1+\frac{1}{\theta}\right)
\end{aligned}
$$




$$
\begin{aligned}
& +\frac{1+\theta}{2}\left(D\left\|\nabla w_{*}\right\|_{L_{2}(\Omega)}^{2}+\alpha\left\|w_{*}\right\|_{L_{2}(\Omega)}^{2}\right) \\
= & \int_{\Omega}\left(u_{*}+v_{*}\right)\left(\ln \left(\frac{\theta M}{\theta+1}\right)-\ln \left(\left\|e^{w_{*}}\right\|_{L_{1}(\Omega)}\right)\right) \mathrm{d} x-M+|\Omega|\left(1+\frac{1}{\theta}\right) \\
& +\frac{1+\theta}{2}\left(D\left\|\nabla w_{*}\right\|_{L_{2}(\Omega)}^{2}+\alpha\left\|w_{*}\right\|_{L_{2}(\Omega)}^{2}\right) \\
= & M \ln \left(\frac{\theta M}{\theta+1}\right)-M \ln \left(\left\|e^{w_{*}}\right\|_{L_{1}(\Omega)}\right)-M+|\Omega|\left(1+\frac{1}{\theta}\right) \\
& +\frac{1+\theta}{2}\left(D\left\|\nabla w_{*}\right\|_{L_{2}(\Omega)}^{2}+\alpha\left\|w_{*}\right\|_{L_{2}(\Omega)}^{2}\right) .
\end{aligned}
$$

As (5.1) and (5.3) imply

$$
w_{*} \geq 0 \quad \text { in } \Omega \quad \text { and } \quad\left\|w_{*}\right\|_{L_{1}(\Omega)}=\frac{M}{\alpha(\theta+1)},
$$

we define

$$
W:=w_{*}-\frac{M}{\alpha(\theta+1)|\Omega|}=w_{*}-\frac{1}{|\Omega|} \int_{\Omega} w_{*}(x) \mathrm{d} x .
$$

Rewriting (5.4) in terms of $W$ leads to

$$
\begin{aligned}
\mathcal{L}\left(u_{*}, v_{*}, w_{*}\right)= & \frac{1+\theta}{2}\left(D\|\nabla W\|_{L_{2}(\Omega)}^{2}+\alpha\|W\|_{L_{2}(\Omega)}^{2}+\alpha|\Omega| \frac{M^{2}}{\alpha^{2}(\theta+1)^{2}|\Omega|^{2}}\right) \\
& -M \ln \left(\left\|e^{W}\right\|_{L_{1}(\Omega)}\right)-\frac{M^{2}}{\alpha(\theta+1)|\Omega|}+M \ln \left(\frac{\theta M}{\theta+1}\right) \\
& -M+|\Omega|\left(1+\frac{1}{\theta}\right) \\
= & \frac{M}{|\Omega|} \mathcal{F}(W)-M \ln (|\Omega|)-\frac{M^{2}}{2 \alpha(\theta+1)|\Omega|}+M \ln \left(\frac{\theta M}{\theta+1}\right) \\
& -M+|\Omega|\left(1+\frac{1}{\theta}\right),
\end{aligned}
$$

where

$$
\mathcal{F}(W):=\frac{(1+\theta)|\Omega|}{2 M}\left(D\|\nabla W\|_{L_{2}(\Omega)}^{2}+\alpha\|W\|_{L_{2}(\Omega)}^{2}\right)-|\Omega| \ln \left(\frac{\left\|e^{W}\right\|_{L_{1}(\Omega)}}{|\Omega|}\right) .
$$

Moreover, in view of (5.3) and (5.5), $W$ is a solution to

$$
-D \Delta W+\alpha W=\frac{M}{(\theta+1)|\Omega|}\left(\frac{|\Omega| e^{W}}{\left\|e^{W}\right\|_{L_{1}(\Omega)}}-1\right) \quad \text { in } \Omega, \quad \nabla W \cdot \mathbf{n}=0 \quad \text { on } \partial \Omega,
$$

along with $\int_{\Omega} W(x) \mathrm{d} x=0$. Hence, due to (5.7), (5.8) along with $M \in(4 \pi(1+\theta) D, \infty) \backslash$ $(4 \pi(1+\theta) D \mathbb{N})$, we are in a position to apply [13, Lemma 3.5] and conclude that there exists $\mu \geq 0$ 
which does not depend on $W$ such that

$$
\mathcal{F}(W) \geq-\mu
$$

Combining the latter with (5.6) completes the proof of assertion (a).

(b) The proof is the same as that of assertion (a), we only use [13, Corollary $3.7 \&$ Remark 3.8] instead of [13, Lemma 3.5] to deduce the lower bound (5.9) for any $M \in(8 \pi(1+\theta) D, \infty)$.

As in $[12,13,29]$, the next step is to show that $\mathcal{L}$ is not bounded from below on the set $\mathcal{I}_{M}$ of initial conditions defined in (1.7) as soon as $M$ exceeds a specific threshold value. The argument given below is however more involved, due to the additional positive term in $\mathcal{L}$.

Proposition 5.2. Let $M>0$.

(a) If $M \in(4 \pi(1+\theta) D, \infty)$, then

$$
\inf _{(u, v, w) \in \mathcal{I}_{M}} \mathcal{L}(u, v, w)=-\infty .
$$

(b) If $\Omega=B_{R}(0)$ for some $R>0$ and $M \in(8 \pi(1+\theta) D$, $\infty)$, then

$$
\inf _{(u, v, w) \in \mathcal{I}_{M, \text { rad }}} \mathcal{L}(u, v, w)=-\infty,
$$

where $\mathcal{I}_{M, \text { rad }}:=\left\{(u, v, w) \in \mathcal{I}_{M}: u, v, w\right.$ are radially symmetric $\}$.

Proof. (a) As $\partial \Omega$ is smooth, upon a translation and a rotation, we may assume without loss of generality that $0 \in \partial \Omega, \mathbf{n}(0)=(0,-1)^{T}$, and that there exist $a_{0} \in(0,1)$ and $\zeta \in C^{2}\left(\left[-a_{0}, a_{0}\right]\right)$ such that we have

$$
\Omega \cap B_{a_{0}}(0)=\left\{x \in B_{a_{0}}(0): x_{2}>\zeta\left(x_{1}\right)\right\} \quad \text { and } \quad \partial \Omega \cap B_{a_{0}}(0)=\left\{x \in B_{a_{0}}(0): x_{2}=\zeta\left(x_{1}\right)\right\} .
$$

We first claim that there is $\bar{\omega} \in C\left(\left[0, a_{0}\right]\right)$ such that

$$
\bar{\omega}(0)=0, \quad \bar{\omega} \geq 0,
$$

and, for all $a \in\left(0, a_{0}\right)$,

$$
\sigma_{a} \subset \Omega \cap B_{a}(0) \subset \Sigma_{a}
$$

where

$$
\begin{aligned}
\sigma_{a} & :=\{x=(r \cos (\omega), r \sin (\omega)): \\
\Sigma_{a} & :=\{x=(r \cos (\omega), r \sin (\omega)): a), \omega \in(\bar{\omega}(a), \pi-\bar{\omega}(a))\}, \\
& : r \in[0, a), \omega \in[0, \pi+\bar{\omega}(a)) \cup(2 \pi-\bar{\omega}(a), 2 \pi)\} .
\end{aligned}
$$

Indeed, in view of $0 \in \partial \Omega$ we have $\zeta(0)=0$, while $\mathbf{n}(0)=(0,-1)^{T}$ implies $\zeta^{\prime}(0)=0$. Hence, with $A:=\left\|\zeta^{\prime \prime}\right\|_{C\left(\left[-a_{0}, a_{0}\right]\right)} / 2 \in[0, \infty)$, a Taylor expansion implies

$$
|\zeta(s)|=\left|\zeta(s)-\zeta(0)-\zeta^{\prime}(0) s\right| \leq A s^{2} \leq A a|s| \quad \text { for all } s \in[-a, a]
$$

and any $a \in\left(0, a_{0}\right]$. Combining these properties of $\Omega$ and $\zeta$, we deduce that

$$
\left\{x \in B_{a}(0): x_{2}>A a\left|x_{1}\right|\right\} \subset \Omega \cap B_{a}(0) \subset\left\{x \in B_{a}(0): x_{2}>-A a\left|x_{1}\right|\right\} .
$$


Hence, (5.10a) and (5.10b) are satisfied for any $a \in\left(0, a_{0}\right)$ with the continuous function $\bar{\omega}(a):=$ $\arctan (A a), a \in\left[0, a_{0}\right]$.

Next, for $\eta \in(0,1)$ and $x \in \Omega$, define

$$
\xi_{\eta}(x):=2 \ln \left(\frac{\eta}{\eta^{2}+\pi|x|^{2}}\right) \quad \text { and } \Xi_{\eta}(x):=\xi_{\eta}(x)-\frac{1}{|\Omega|} \int_{\Omega} \xi_{\eta}(y) \mathrm{d} y .
$$

Clearly, $\xi_{\eta}$ belongs to $W_{3}^{2}(\Omega)$ and, for $(x, \eta) \in \Omega \times(0,1)$,

$$
\begin{aligned}
\nabla \xi_{\eta}(x) & =-\frac{4 \pi x}{\eta^{2}+\pi|x|^{2}} \\
D^{2} \xi_{\eta}(x) & =-\frac{4 \pi}{\eta^{2}+\pi|x|^{2}} \mathrm{id}+\frac{8 \pi^{2}}{\left(\eta^{2}+\pi|x|^{2}\right)^{2}} x \otimes x \\
-\Delta \xi_{\eta}(x) & =\frac{8 \pi \eta^{2}}{\left(\eta^{2}+\pi|x|^{2}\right)^{2}}=8 \pi e^{\xi_{\eta}(x)} .
\end{aligned}
$$

In view of (5.10a) and $M>4 \pi(1+\theta) D>4 \pi D$, we next fix $a \in\left(0, a_{0}\right)$ and $\eta_{0} \in(0,1)$ sufficiently small such that

$$
\begin{aligned}
& \bar{\omega}(a)<\max \left\{\frac{\pi}{4}, \frac{M-4 \pi D}{32 D}\right\}, \\
& \eta_{0}^{2}+\pi a^{2}<1, \\
& \eta_{0}^{2}<\frac{M-4 \pi D}{32 D|\Omega|} \pi a^{4},
\end{aligned}
$$

and derive additional estimates on $\xi_{\eta}$ for $\eta \in\left(0, \eta_{0}\right)$. First, by $(5.10 \mathrm{~b})$,

$$
\begin{aligned}
\left\|e^{\xi_{\eta}}\right\|_{L_{1}(\Omega)} & =\int_{\Omega \cap B_{a}(0)} \frac{\eta^{2}}{\left(\eta^{2}+\pi|x|^{2}\right)^{2}} \mathrm{~d} x+\int_{\Omega \cap B_{a}(0)^{c}} \frac{\eta^{2}}{\left(\eta^{2}+\pi|x|^{2}\right)^{2}} \mathrm{~d} x \\
& \leq \int_{\Sigma_{a}} \frac{\eta^{2}}{\left(\eta^{2}+\pi|x|^{2}\right)^{2}} \mathrm{~d} x+\int_{\Omega \cap B_{a}(0)^{c}} \frac{\eta^{2}}{\left(\eta^{2}+\pi a^{2}\right)^{2}} \mathrm{~d} x \\
& \leq \frac{\pi+2 \bar{\omega}(a)}{2 \pi}\left[-\frac{\eta^{2}}{\eta^{2}+\pi r^{2}}\right]_{r=0}^{r=a}+\frac{\eta^{2}|\Omega|}{\pi^{2} a^{4}} \\
& \leq \frac{1}{2}+\frac{\bar{\omega}(a)}{\pi}+\frac{\eta_{0}^{2}|\Omega|}{\pi^{2} a^{4}},
\end{aligned}
$$

and

$$
\begin{aligned}
\left\|e^{\xi_{\eta}}\right\|_{L_{1}(\Omega)} & \geq \int_{\Omega \cap B_{a}(0)} \frac{\eta^{2}}{\left(\eta^{2}+\pi|x|^{2}\right)^{2}} \mathrm{~d} x \geq \int_{\sigma_{a}} \frac{\eta^{2}}{\left(\eta^{2}+\pi|x|^{2}\right)^{2}} \mathrm{~d} x \\
& =\frac{\pi-2 \bar{\omega}(a)}{2 \pi}\left[-\frac{\eta^{2}}{\eta^{2}+\pi r^{2}}\right]_{r=0}^{r=a}=\frac{\pi-2 \bar{\omega}(a)}{2 \pi} \frac{\pi a^{2}}{\eta^{2}+\pi a^{2}}
\end{aligned}
$$




$$
\geq \frac{\pi-2 \bar{\omega}(a)}{2 \pi} \frac{\pi a^{2}}{\eta_{0}^{2}+\pi a^{2}}
$$

Hence, using (5.12),

$$
\frac{\pi a^{2}}{4}<\left\|e^{\xi_{\eta}}\right\|_{L_{1}(\Omega)}<\frac{1}{2}+\frac{M-4 \pi D}{16 \pi D}=\frac{M+4 \pi D}{16 \pi D} .
$$

We next turn to $\Xi_{\eta}$ and first derive a lower bound for $\eta \in\left(0, \eta_{0}\right)$. To this end, we compute

$$
\begin{aligned}
I_{\eta} & :=\frac{2}{|\Omega|} \int_{\Omega} \ln \left(\eta^{2}+\pi|y|^{2}\right) \mathrm{d} y \\
& =\frac{2}{|\Omega|} \int_{\Omega \cap B_{a}(0)} \ln \left(\eta^{2}+\pi|y|^{2}\right) \mathrm{d} y+\frac{2}{|\Omega|} \int_{\Omega \cap B_{a}(0)^{c}} \ln \left(\eta^{2}+\pi|y|^{2}\right) \mathrm{d} y \\
& \geq \frac{2}{|\Omega|} \int_{\Omega \cap B_{a}(0)} \ln \left(\eta^{2}+\pi|y|^{2}\right) \mathrm{d} y+\frac{2}{|\Omega|} \int_{\Omega \cap B_{a}(0)^{c}} \ln \left(\pi a^{2}\right) \mathrm{d} y .
\end{aligned}
$$

Since $\eta^{2}+\pi|y|^{2} \leq \eta_{0}^{2}+\pi a^{2}<1$ for $(\eta, y) \in\left(0, \eta_{0}\right) \times \Sigma_{a}$ by (5.12b), we infer from (5.10b) and (5.12a) that

$$
\begin{aligned}
I_{\eta} & \geq \frac{2}{|\Omega|} \int_{\Sigma_{a}} \ln \left(\eta^{2}+\pi|y|^{2}\right) \mathrm{d} y+\frac{4}{|\Omega|} \int_{\Omega \cap B_{a}(0)^{c}} \ln a \mathrm{~d} y \\
& \geq \frac{\pi+2 \bar{\omega}(a)}{\pi|\Omega|}\left[\left(\eta^{2}+\pi r^{2}\right) \ln \left(\eta^{2}+\pi r^{2}\right)-\eta^{2}-\pi r^{2}+1\right]_{r=0}^{r=a}+\frac{4}{|\Omega|} \int_{\Omega} \ln a \mathrm{~d} y \\
& \geq \frac{\pi+2 \bar{\omega}(a)}{\pi|\Omega|}\left(-2 \eta^{2} \ln \eta+\eta^{2}-1\right)-4|\ln a| \geq-\frac{\pi+2 \bar{\omega}(a)}{\pi|\Omega|}-4|\ln a| \\
& \geq-\frac{1}{|\Omega|}\left(1+\frac{M}{16 \pi D}\right)-4|\ln a| .
\end{aligned}
$$

Consequently, for $x \in \Omega$,

$$
\Xi_{\eta}(x)=-2 \ln \left(\eta^{2}+\pi|x|^{2}\right)+I_{\eta} \geq-\nu_{1},
$$

with $R:=\operatorname{diam}(\Omega) / 2$ and

$$
\nu_{1}:=2 \ln \left(1+4 \pi R^{2}\right)+\frac{1}{|\Omega|}\left(1+\frac{M}{16 \pi D}\right)+4|\ln a| .
$$

Finally,

$$
\begin{aligned}
\left\|\Xi_{\eta}\right\|_{L_{2}(\Omega)}^{2} & =\int_{\Omega}\left(-2 \ln \left(\eta^{2}+\pi|x|^{2}\right)+I_{\eta}\right)^{2} \mathrm{~d} x=4 \int_{\Omega}\left(\ln \left(\eta^{2}+\pi|x|^{2}\right)\right)^{2} \mathrm{~d} x-|\Omega| I_{\eta}^{2} \\
& \leq 4 \int_{B_{2 R}(0)}\left(\ln \left(\eta^{2}+\pi|x|^{2}\right)\right)^{2} \mathrm{~d} x=8 \pi \int_{0}^{2 R} r\left(\ln \left(\eta^{2}+\pi r^{2}\right)\right)^{2} \mathrm{~d} r \\
& =4\left[\left(\eta^{2}+\pi r^{2}\right)\left(\ln \left(\eta^{2}+\pi r^{2}\right)\right)^{2}-2\left(\eta^{2}+\pi r^{2}\right) \ln \left(\eta^{2}+\pi r^{2}\right)+2\left(\eta^{2}+\pi r^{2}\right)\right]_{r=0}^{r=2 R} \\
& \leq \nu_{2}^{2},
\end{aligned}
$$


where

$$
\nu_{2}^{2}:=4\left[\left(1+4 \pi R^{2}\right)\left(\ln \left(1+4 \pi R^{2}\right)\right)^{2}-2\left(1+4 \pi R^{2}\right) \ln \left(1+4 \pi R^{2}\right)+2\left(1+4 \pi R^{2}\right)\right] .
$$

Now, for $\eta \in\left(0, \eta_{0}\right)$, we set

$$
u_{\eta}:=U_{\eta} \frac{e^{\xi_{\eta}}}{\left\|e^{\xi_{\eta}}\right\|_{L_{1}(\Omega)}}, \quad v_{\eta}:=V_{\eta} \frac{e^{\xi_{\eta}}}{\left\|e^{\xi_{\eta}}\right\|_{L_{1}(\Omega)}}, \quad w_{\eta}=\Xi_{\eta}+\nu_{1},
$$

with

$$
U_{\eta}:=M-8 \pi D\left\|e^{\xi_{\eta}}\right\|_{L_{1}(\Omega)}, \quad V_{\eta}:=8 \pi D\left\|e^{\xi_{\eta}}\right\|_{L_{1}(\Omega)} .
$$

We first observe that (5.13) and the lower bound on $M$ guarantee that

$$
U_{\eta}=\left\|u_{\eta}\right\|_{L_{1}(\Omega)} \in[2 \pi \theta D, M] \text { and } \quad V_{\eta}=\left\|v_{\eta}\right\|_{L_{1}(\Omega)} \in\left[2 \pi^{2} a^{2} D, \frac{2+\theta}{2(1+\theta)} M\right],
$$

while $w_{\eta} \geq 0$ by $(5.14)$, so that the triple $\left(u_{\eta}, v_{\eta}, w_{\eta}\right)$ defined in (5.16) belongs to $\mathcal{I}_{M}$. Also,

Next, on the one hand,

$$
u_{\eta}=U_{\eta} \frac{e^{w_{\eta}}}{\left\|e^{w_{\eta}}\right\|_{L_{1}(\Omega)}} \text { and } v_{\eta}=V_{\eta} \frac{e^{w_{\eta}}}{\left\|e^{w_{\eta}}\right\|_{L_{1}(\Omega)}} .
$$

$$
\begin{aligned}
\int_{\Omega} u_{\eta} \ln u_{\eta} \mathrm{d} x & =\ln \left(U_{\eta}\right) \int_{\Omega} u_{\eta} \mathrm{d} x+\int_{\Omega} u_{\eta} w_{\eta} \mathrm{d} x-\ln \left(\left\|e^{w_{\eta}}\right\|_{L_{1}(\Omega)}\right) \int_{\Omega} u_{\eta} \mathrm{d} x \\
& =\int_{\Omega} u_{\eta} w_{\eta} \mathrm{d} x+U_{\eta} \ln \left(U_{\eta}\right)-U_{\eta} \ln \left(\left\|e^{w_{\eta}}\right\|_{L_{1}(\Omega)}\right)
\end{aligned}
$$

and

$$
\begin{aligned}
\int_{\Omega} v_{\eta} \ln \left(\theta v_{\eta}\right) \mathrm{d} x & =\ln \left(\theta V_{\eta}\right) \int_{\Omega} v_{\eta} \mathrm{d} x+\int_{\Omega} v_{\eta} w_{\eta} \mathrm{d} x-\ln \left(\left\|e^{w_{\eta}}\right\|_{L_{1}(\Omega)}\right) \int_{\Omega} v_{\eta} \mathrm{d} x \\
& =\int_{\Omega} v_{\eta} w_{\eta} \mathrm{d} x+V_{\eta} \ln \left(\theta V_{\eta}\right)-V_{\eta} \ln \left(\left\|e^{w_{\eta}}\right\|_{L_{1}(\Omega)}\right) .
\end{aligned}
$$

On the other hand, by (5.11), (5.15), and (5.16),

$$
\begin{aligned}
\left\|D \Delta w_{\eta}-\alpha w_{\eta}+v_{\eta}\right\|_{L_{2}(\Omega)}^{2} & =\left\|D \Delta \xi_{\eta}+V_{\eta} \frac{e^{\xi_{\eta}}}{\left\|e^{\xi_{\eta}}\right\|_{L_{1}(\Omega)}}-\alpha \Xi_{\eta}-\alpha \nu_{1}\right\|_{L_{2}(\Omega)}^{2} \\
& =\left\|D \Delta \xi_{\eta}+8 \pi D e^{\xi_{\eta}}-\alpha \Xi_{\eta}-\alpha \nu_{1}\right\|_{L_{2}(\Omega)}^{2} \\
& =\alpha^{2}\left\|\Xi_{\eta}+\nu_{1}\right\|_{L_{2}(\Omega)}^{2} \leq 2 \alpha^{2}\left(\left\|\Xi_{\eta}\right\|_{L_{2}(\Omega)}^{2}+|\Omega| \nu_{1}^{2}\right) \\
& \leq 2 \nu_{3}^{2}:=2 \alpha^{2}\left(\nu_{2}^{2}+|\Omega| \nu_{1}^{2}\right) .
\end{aligned}
$$

We then infer from (5.18), (5.19), and (5.20) that

$$
\mathcal{L}\left(u_{\eta}, v_{\eta}, w_{\eta}\right)=\int_{\Omega}\left(u_{\eta} \ln u_{\eta}-u_{\eta}+1+v_{\eta} \ln \left(\theta v_{\eta}\right)-v_{\eta}+\frac{1}{\theta}-\left(u_{\eta}+v_{\eta}\right) w_{\eta}\right) \mathrm{d} x
$$




$$
\begin{aligned}
& +\frac{1+\theta}{2}\left(D\left\|\nabla w_{\eta}\right\|_{L_{2}(\Omega)}^{2}+\alpha\left\|w_{\eta}\right\|_{L_{2}(\Omega)}^{2}\right)+\frac{1}{2}\left\|D \Delta w_{\eta}-\alpha w_{\eta}+v_{\eta}\right\|_{L_{2}(\Omega)}^{2} \\
\leq & U_{\eta} \ln \left(U_{\eta}\right)-U_{\eta}+|\Omega|+V_{\eta} \ln \left(\theta V_{\eta}\right)-V_{\eta}+\frac{|\Omega|}{\theta}-\left(U_{\eta}+V_{\eta}\right) \ln \left(\left\|e^{w_{\eta}}\right\|_{L_{1}(\Omega)}\right) \\
& +\frac{1+\theta}{2}\left(D\left\|\nabla \Xi_{\eta}\right\|_{L_{2}(\Omega)}^{2}+\alpha\left\|\Xi_{\eta}+\nu_{1}\right\|_{L_{2}(\Omega)}^{2}\right)+\nu_{3}^{2} \\
= & U_{\eta} \ln \left(U_{\eta}\right)-U_{\eta}+|\Omega|+V_{\eta} \ln \left(\theta V_{\eta}\right)-V_{\eta}+\frac{|\Omega|}{\theta}-M \ln \left(\left\|e^{\Xi_{\eta}}\right\|_{L_{1}(\Omega)}\right)-M \nu_{1} \\
& +\frac{1+\theta}{2}\left(D\left\|\nabla \Xi_{\eta}\right\|_{L_{2}(\Omega)}^{2}+\alpha\left\|\Xi_{\eta}\right\|_{L_{2}(\Omega)}^{2}+\alpha|\Omega| \nu_{1}^{2}\right)+\nu_{3}^{2} \\
= & \frac{M}{|\Omega|} \mathcal{F}\left(\Xi_{\eta}\right)+\mathcal{R}_{\eta},
\end{aligned}
$$

where

$$
\mathcal{F}\left(\Xi_{\eta}\right)=\frac{(1+\theta)|\Omega|}{2 M}\left(D\left\|\nabla \Xi_{\eta}\right\|_{L_{2}(\Omega)}^{2}+\alpha\left\|\Xi_{\eta}\right\|_{L_{2}(\Omega)}^{2}\right)-|\Omega| \ln \left(\frac{\left\|e^{\Xi_{\eta}}\right\|_{L_{1}(\Omega)}}{|\Omega|}\right),
$$

see (5.7), and

$$
\begin{aligned}
\mathcal{R}_{\eta}:= & -M \ln (|\Omega|)+U_{\eta} \ln \left(U_{\eta}\right)-U_{\eta}+|\Omega|+V_{\eta} \ln \left(\theta V_{\eta}\right)-V_{\eta}+\frac{|\Omega|}{\theta} \\
& -M \nu_{1}+\frac{(1+\theta) \alpha|\Omega|}{2} \nu_{1}^{2}+\nu_{3}^{2} .
\end{aligned}
$$

According to [13, Section 3],

$$
\lim _{\eta \rightarrow 0} \mathcal{F}\left(\Xi_{\eta}\right)=-\infty
$$

while (5.17) ensures that $\sup _{\eta \in\left(0, \eta_{0}\right)} \mathcal{R}_{\eta}<\infty$. In view of these properties, it readily follows from (5.21) that $\mathcal{L}\left(u_{\eta}, v_{\eta}, w_{\eta}\right) \rightarrow-\infty$ as $\eta \rightarrow 0$, as claimed.

(b) We recall that $\Omega=B_{R}(0)$ in that case. As above, for $\eta \in(0,1)$ and $x \in \Omega$, we define

$$
\xi_{\eta}(x):=2 \ln \left(\frac{\eta}{\eta^{2}+\pi|x|^{2}}\right) \quad \text { and } \quad \Xi_{\eta}(x):=\xi_{\eta}(x)-\frac{1}{|\Omega|} \int_{\Omega} \xi_{\eta}(y) \mathrm{d} y .
$$

Then $\xi_{\eta} \in W_{3}^{2}(\Omega)$ and

$$
\begin{aligned}
\left\|e^{\xi_{\eta}}\right\|_{L_{1}(\Omega)} & =\int_{\Omega} \frac{\eta^{2}}{\left(\eta^{2}+\pi|x|^{2}\right)^{2}} \mathrm{~d} x=\eta^{2} \int_{0}^{R} \frac{2 \pi r}{\left(\eta^{2}+\pi r^{2}\right)^{2}} \mathrm{~d} r \\
& =-\left[\frac{\eta^{2}}{\eta^{2}+\pi r^{2}}\right]_{r=0}^{r=R}=\frac{\pi R^{2}}{\eta^{2}+\pi R^{2}} .
\end{aligned}
$$

Hence,

$$
\frac{\pi R^{2}}{1+\pi R^{2}} \leq\left\|e^{\xi_{\eta}}\right\|_{L_{1}(\Omega)} \leq 1
$$


Furthermore,

so that

$$
\begin{aligned}
I_{\eta} & :=\frac{2}{\pi R^{2}} \int_{\Omega} \ln \left(\eta^{2}+\pi|y|^{2}\right) \mathrm{d} y=\frac{4}{R^{2}} \int_{0}^{R} \ln \left(\eta^{2}+\pi r^{2}\right) r \mathrm{~d} r \\
& =\frac{2}{\pi R^{2}}\left[\left(\eta^{2}+\pi r^{2}\right) \ln \left(\eta^{2}+\pi r^{2}\right)-\left(\eta^{2}+\pi r^{2}\right)+1\right]_{r=0}^{r=R} \\
& \geq \frac{2}{\pi R^{2}}\left(-2 \eta^{2} \ln \eta+\eta^{2}-1\right) \geq-\frac{2}{\pi R^{2}}
\end{aligned}
$$

$$
\Xi_{\eta}(x) \geq-2 \ln \left(\eta^{2}+\pi R^{2}\right)+I_{\eta} \geq-\nu_{4}:=-2 \ln \left(1+\pi R^{2}\right)-\frac{2}{\pi R^{2}}
$$

Finally,

$$
\begin{aligned}
\left\|\Xi_{\eta}\right\|_{L_{2}(\Omega)}^{2} & =\int_{\Omega}\left[-2 \ln \left(\eta^{2}+\pi|x|^{2}\right)+I_{\eta}\right]^{2} \mathrm{~d} x=4 \int_{\Omega}\left(\ln \left(\eta^{2}+\pi|x|^{2}\right)\right)^{2} \mathrm{~d} x-|\Omega| I_{\eta}^{2} \\
& \leq 4\left[\left(\eta^{2}+\pi r^{2}\right)\left[\ln \left(\eta^{2}+\pi r^{2}\right)\right]^{2}-2\left(\eta^{2}+\pi r^{2}\right) \ln \left(\eta^{2}+\pi r^{2}\right)+2\left(\eta^{2}+\pi r^{2}\right)\right]_{r=0}^{r=R} \\
& \leq \nu_{5}^{2}
\end{aligned}
$$

where

$$
\nu_{5}^{2}:=4\left[\left(1^{2}+\pi R^{2}\right)\left[\ln \left(1+\pi R^{2}\right)\right]^{2}-2\left(1+\pi R^{2}\right) \ln \left(1+\pi R^{2}\right)+2\left(1+\pi R^{2}\right)\right] .
$$

Now, as above, we set

$$
u_{\eta}:=U_{\eta} \frac{e^{\xi_{\eta}}}{\left\|e^{\xi_{\eta}}\right\|_{L_{1}(\Omega)}}, \quad v_{\eta}:=V_{\eta} \frac{e^{\xi_{\eta}}}{\left\|e^{\xi_{\eta}}\right\|_{L_{1}(\Omega)}}, w_{\eta}=\Xi_{\eta}+\nu_{4},
$$

with

$$
U_{\eta}:=M-8 \pi D\left\|e^{\xi_{\eta}}\right\|_{L_{1}(\Omega)}, \quad V_{\eta}:=8 \pi D\left\|e^{\xi_{\eta}}\right\|_{L_{1}(\Omega)},
$$

and deduce from (5.22) and the lower bound on $M$ that

$$
U_{\eta} \in\left[\frac{\theta M}{1+\theta}, M\right] \text { and } V_{\eta} \in\left[\frac{8 \pi^{2} R^{2} D}{1+\pi R^{2}}, \frac{M}{1+\theta}\right] .
$$

In particular, owing to $(5.26),(5.27)$, and the regularity of $\xi_{\eta}$, the triple $\left(u_{\eta}, v_{\eta}, w_{\eta}\right)$ belongs to $\mathcal{I}_{M \text {, rad }}$ for all $\eta \in(0,1)$. We next compute $\mathcal{L}\left(u_{\eta}, v_{\eta}, w_{\eta}\right)$ as in the previous case and argue as in the proof of (5.21) with the help of $(5.11),(5.24)$, and (5.25) to obtain that

$$
\mathcal{L}\left(u_{\eta}, v_{\eta}, w_{\eta}\right) \leq \frac{M}{|\Omega|} \mathcal{F}\left(\Xi_{\eta}\right)+\mathcal{R}_{\eta}
$$

where

$$
\mathcal{F}\left(\Xi_{\eta}\right)=\frac{(1+\theta)|\Omega|}{2 M}\left(D\left\|\nabla \Xi_{\eta}\right\|_{L_{2}(\Omega)}^{2}+\alpha\left\|\Xi_{\eta}\right\|_{L_{2}(\Omega)}^{2}\right)-|\Omega| \ln \left(\frac{\left\|e^{\Xi_{\eta}}\right\|_{L_{1}(\Omega)}}{|\Omega|}\right)
$$


as before and

$$
\begin{aligned}
\mathcal{R}_{\eta}:= & -M \ln (|\Omega|)+U_{\eta} \ln \left(U_{\eta}\right)-U_{\eta}+|\Omega|+V_{\eta} \ln \left(\theta V_{\eta}\right)-V_{\eta}+\frac{|\Omega|}{\theta} \\
& -M \nu_{4}+\frac{(1+\theta) \alpha|\Omega|}{2} \nu_{4}^{2}+\alpha^{2}\left(\nu_{5}^{2}+|\Omega| \nu_{4}^{2}\right) .
\end{aligned}
$$

According to [12, Lemma 2],

$$
\lim _{\eta \rightarrow 0} \mathcal{F}\left(\Xi_{\eta}\right)=-\infty
$$

while (5.27) implies that $\sup _{\eta \in(0,1)} \mathcal{R}_{\eta}<\infty$. It then readily follows from (5.28) and the above properties that $\mathcal{L}\left(u_{\eta}, v_{\eta}, w_{\eta}\right) \longrightarrow-\infty$ as $\eta \rightarrow 0$ and the proof of (b) is complete.

Proof of Theorem 1.3. (a) Gathering the outcome of Proposition 2.2, Proposition 5.1 (a) and Proposition 5.2 (a), we argue as in $[12,13,17,29]$, see also [15], to conclude that, for $M \in(4 \pi(1+\theta) D, \infty) \backslash$ $(4 \pi(1+\theta) D \mathbb{N})$, there are initial conditions in $\mathcal{I}_{M}$ for which the first component of the corresponding solution to (1.1)-(1.2) cannot be bounded in $L_{\infty}((0, \infty) \times \Omega)$ and thus infringes (2.6).

(b) In that case, combining Proposition 2.2, Proposition 5.1 (b) and Proposition 5.2 (b) with the above mentioned references leads to the claim.

\section{REFERENCES}

[1] H. Amann, Dual semigroups and second order linear elliptic boundary value problems, Israel J. Math., 45 (1983), pp. 225-254.

[2] —_ Highly degenerate quasilinear parabolic systems, Ann. Scuola Norm. Sup. Pisa Cl. Sci. (4), 18 (1991), pp. $135-166$.

[3] - Nonhomogeneous linear and quasilinear elliptic and parabolic boundary value problems, in Function spaces, differential operators and nonlinear analysis (Friedrichroda, 1992), vol. 133 of Teubner-Texte Math., Teubner, Stuttgart, 1993, pp. 9-126.

[4] _ Linear and quasilinear parabolic problems. Vol. I, Abstract linear theory, vol. 89 of Monographs in Mathematics, Birkhäuser Boston, Inc., Boston, MA, 1995.

[5] P. Biler, W. Hebisch, And T. Nadzieja, The Debye system: existence and large time behavior of solutions, Nonlinear Anal., 23 (1994), pp. 1189-1209.

[6] P. Biler And T. NADziEJA, Existence and nonexistence of solutions for a model of gravitational interaction of particles. I, Colloq. Math., 66 (1993), pp. 319-334.

[7] S.-Y. A. Chang And P. C. YAng, Conformal deformation of metrics on $S^{2}$, J. Differential Geom., 27 (1988), pp. 259-296.

[8] T. CieŚlak and C. Stinner, New critical exponents in a fully parabolic quasilinear Keller-Segel system and applications to volume filling models, J. Differential Equations, 258 (2015), pp. 2080-2113.

[9] J. Deneubourg, Application de l'ordre par fluctuations à la description de certaines étapes de la construction du nid chez les termites, Insectes Sociaux, 24 (1977), pp. 117-130.

[10] H. Gajewski And K. Zacharias, Global behaviour of a reaction-diffusion system modelling chemotaxis, Math. Nachr., 195 (1998), pp. 77-114.

[11] D. Horstmann, The nonsymmetric case of the Keller-Segel model in chemotaxis: some recent results, NoDEA Nonlinear Differential Equations Appl., 8 (2001), pp. 399-423.

[12] _ - On the existence of radially symmetric blow-up solutions for the Keller-Segel model, J. Math. Biol., 44 (2002), pp. 463-478. 
[13] D. Horstmann And G. Wang, Blow-up in a chemotaxis model without symmetry assumptions, European J. Appl. Math., 12 (2001), pp. 159-177.

[14] B. Hu AND Y. TAO, To the exclusion of blow-up in a three-dimensional chemotaxis-growth model with indirect attractant production, Math. Models Methods Appl. Sci., 26 (2016), pp. 2111-2128.

[15] S. Ishida And T. Yoкота, Blow-up in finite or infinite time for quasilinear degenerate Keller-Segel systems of parabolic-parabolic type, Discrete Contin. Dyn. Syst. Ser. B, 18 (2013), pp. 2569-2596.

[16] E. F. Keller And L. A. Segel, Initiation of slime mold aggregation viewed as an instability, J. Theoret. Biol., 26 (1970), pp. 399-415.

[17] Ph. Laurençot, Global bounded and unbounded solutions to a chemotaxis system with indirect signal production, Discrete Contin. Dyn. Syst. Ser. B, 24 (2019), pp. 6419-6444.

[18] X. LI, Global existence and boundedness of a chemotaxis model with indirect production and general kinetic function, Z. Angew. Math. Phys., 71 (2020). Paper No. 117, 22 pp.

[19] Y. Li And J. LAnkeit, Boundedness in a chemotaxis-haptotaxis model with nonlinear diffusion, Nonlinearity, 29 (2016), pp. 1564-1595.

[20] J. A. Logan, P. White, B. J. Bentz, and J. A. Powell, Model analysis of spatial patterns in mountain pine beetle outbreaks, Theor. Popul. Biol., 53 (1998), pp. 236-255.

[21] T. Nagai, T. Senba, And K. Yoshida, Application of the Trudinger-Moser inequality to a parabolic system of chemotaxis, Funkcial. Ekvac., 40 (1997), pp. 411-433.

[22] E. Nakaguchi, K. Noda, K. Osaki, And K. Uemichi, Global attractor for a two-dimensional chemotaxis system with linear degradation and indirect signal production, Jpn. J. Ind. Appl. Math., 37 (2020), pp. 49-80.

[23] K. J. PAinter, Mathematical models for chemotaxis and their applications in self-organisation phenomena, J. Theoret. Biol., 481 (2019), pp. 162-182.

[24] K. J. Painter, W. Ho, And D. J. Headon, A chemotaxis model of feather primordia pattern formation during avian development, J. Theoret. Biol., 437 (2018), pp. 225-238.

[25] A. PAZY, Semigroups of linear operators and applications to partial differential equations, vol. 44 of Applied Mathematical Sciences, Springer-Verlag, New York, 1983.

[26] J. A. Powell, T. McMillen, And P. White, Connecting a chemotactic model for mass attack to a rapid integro-difference emulation strategy, SIAM J. Appl. Math., 59 (1999), pp. 547-572.

[27] S. Ramakrishnan, T. Laurent, M. Kumar, and A. L. Bertozzi, Spatiotemporal chemotactic model for ant foraging, Modern Phys. Lett. B, 28 (2014), p. 1450238.

[28] J. D. Ravichandar, A. G. Bower, A. A. Julius, and C. H. Collins, Transcriptional control of motility enables directional movement of escherichia coli in a signal gradient, Scientific Reports, 7 (2017), p. 8959.

[29] T. Senba And T. Suzuki, Parabolic system of chemotaxis: blowup in a finite and the infinite time, Methods Appl. Anal., 8 (2001), pp. 349-367. IMS Workshop on Reaction-Diffusion Systems (Shatin, 1999).

[30] C. Stinner, C. Surulescu, and A. UAtay, Global existence for a go-or-grow multiscale model for tumor invasion with therapy, Math. Models Methods Appl. Sci., 26 (2016), pp. 2163-2201.

[31] S. Strohm, R. C. Tyson, And J. A. Powell, Pattern formation in a model for mountain pine beetle dispersal: linking model predictions to data, Bull. Math. Biol., 75 (2013), pp. 1778-1797.

[32] N. Tania, B. Vanderlei, J. P. Heath, and L. Edelstein-Keshet, Role of social interactions in dynamic patterns of resource patches and forager aggregation, Proc. Natl. Acad. Sci. USA, 109 (2012), pp. 11228-11233.

[33] Y. TaO And M. Winkler, Boundedness in a quasilinear parabolic-parabolic Keller-Segel system with subcritical sensitivity, J. Differential Equations, 252 (2012), pp. 692-715.

[34] — Critical mass for infinite-time aggregation in a chemotaxis model with indirect signal production, J. Eur. Math. Soc. (JEMS), 19 (2017), pp. 3641-3678.

[35] Y. TIAn, D. LI, AND C. Mu, Stabilization in three-dimensional chemotaxis-growth model with indirect attractant production, C. R. Math. Acad. Sci. Paris, 357 (2019), pp. 513-519. 
[36] P. White And J. Powell, Spatial invasion of pine beetles into lodgepole forests: a numerical approach, SIAM J. Sci. Comput., 20 (1998), pp. 164-184.

Institut de Mathématiques de Toulouse, UMR 5219, Université de Toulouse, CNRS, F-31062 Toulouse Cedex 9, France

Email address: laurenco@math.univ-toulouse.fr

Technische Universität Darmstadt, Fachbereich Mathematik, Schlossgartenstr. 7, D-64289 DarmSTADT, Germany

Email address: stinner@mathematik.tu-darmstadt.de 